\title{
Testing warm Comptonization models for the origin of the soft $X$-ray excess in AGNs
}

\author{
P.-O. Petrucci ${ }^{1}$, F. Ursini ${ }^{2}$, A. De Rosa ${ }^{3}$, S. Bianchi ${ }^{4}$, M. Cappi $^{2}$, G. Matt ${ }^{4}$, M. Dadina ${ }^{2}$, and J. Malzac ${ }^{5}$ \\ ${ }^{1}$ Univ. Grenoble Alpes, CNRS, IPAG, 38000 Grenoble, France \\ e-mail: pierre-olivier . petrucci@univ-grenoble-alpes.fr \\ 2 INAF-IASF Bologna, Via Gobetti 101, 40129 Bologna, Italy \\ ${ }^{3}$ INAF/Istituto di Astrofisica e Planetologie Spaziali, Via Fosso del Cavaliere, 00133 Roma, Italy \\ ${ }^{4}$ Dipartimento di Matematica e Fisica, Università degli Studi Roma Tre, Via della Vasca Navale 84, 00146 Roma, Italy \\ ${ }^{5}$ IRAP, Université de toulouse, CNRS, UPS, CNES, Toulouse, France
}

Received 17 July 2017 / Accepted 13 October 2017

\begin{abstract}
The X-ray spectra of many active galactic nuclei (AGNs) show a soft X-ray excess below $1-2 \mathrm{keV}$ on top of the extrapolated highenergy power law. The origin of this component is uncertain. It could be a signature of relativistically blurred, ionized reflection or the high-energy tail of thermal Comptonization in a warm $(k T \sim 1 \mathrm{keV})$, optically thick $(\tau \simeq 10-20)$ corona producing the optical/UV to soft X-ray emission. The purpose of the present paper is to test the warm corona model on a statistically significant sample of unabsorbed, radio-quiet AGNs with XMM-Newton archival data, providing simultaneous optical/UV and X-ray coverage. The sample has 22 objects and 100 observations. We use two thermal Comptonization components to fit the broadband spectra, one for the warm corona emission and one for the high-energy continuum. In the optical/UV, we also include the reddening, the small blue bump, and the Galactic extinction. In the X-rays, we include a warm absorber and a neutral reflection. The model gives a good fit (reduced $\left.\chi^{2}<1.5\right)$ to more than $90 \%$ of the sample. We find the temperature of the warm corona to be uniformly distributed in the $0.1-1 \mathrm{keV}$ range, while the optical depth is in the range $\sim 10-40$. These values are consistent with a warm corona covering a large fraction of a quasi-passive accretion disk, i.e., that mostly reprocesses the warm corona emission. The disk intrinsic emission represents no more than $20 \%$ of the disk total emission. According to this interpretation, most of the accretion power would be released in the upper layers of the accretion flow.
\end{abstract}

Key words. galaxies: active - galaxies: Seyfert - X-rays: galaxies

\section{Introduction}

The origin of the soft X-ray excess is a long-standing issue in our understanding of the active galactic nuclei (AGNs) X-ray emission. This excess, above the extrapolation to low energy of the high-energy ( $>2 \mathrm{keV}$ ) continuum power-law fit, was discovered in the 1980s thanks to EXOSAT (Arnaud et al. 1985) and since then has been observed in a large fraction of AGNs (e.g., Walter \& Fink 1993; Page et al. 2004; Gierlinski \& Done 2004; Crummy et al. 2006).

It was realized that its characteristic temperature (e.g., when fitted with a simple blackbody) was remarkably constant over a wide range of AGN luminosities and black hole masses (e.g., Walter \& Fink 1993; Gierlinski \& Done 2004; Bianchi et al. 2009; Crummy et al. 2006), favoring an origin through atomic processes instead of purely continuum emission. Different models, assuming either blurred ionized reflection or thermal Comptonization in an optically thick $(\tau>1)$ and warm $(k T \sim 1 \mathrm{keV})$ plasma, have been proposed to fit this component and both assumptions give acceptable results (Crummy et al. 2006; Magdziarz et al. 1998; Done et al. 2012; Jin et al. 2012b).

The thermal Comptonization modeling of the soft X-ray excess has been carefully tested with the data set from the large broadband campaign on Mrk 509 (Kaastra et al. 2011; Petrucci et al. 2013, P13 hereafter). This campaign is still unique in terms of duration, energy coverage, and number of observations involved. At the core of this program are ten observations with XMM-Newton of approximately $60 \mathrm{ks}$ each spaced by four days. INTEGRAL observations were obtained simultaneously, extending the energy coverage up to the hard X-rays. A strong correlation between the UV and the soft X-ray $(<0.5 \mathrm{keV})$ flux was observed during this campaign, while no correlation was found between the UV and the hard (>3 keV) X-rays (see Mehdipour et al. 2011). This suggested that the UV and soft $\mathrm{X}$-ray emissions were produced by the same spectral component, an interpretation that was supported by a Principal Component Analysis (P13). Combined with the absence of a broad iron line component, which would have been expected if blurred reflection had been present in this source, the thermal Comptonization appeared naturally to be the most plausible scenario.

The data were then fitted with a two-coronae model, which assumes two Comptonization model components, one for the UV-Soft X, the so-called warm corona, and one for the hard X, the so-called hot corona (see P13 for more details). Warm absorption and neutral reflection were also included in the fit procedure. The model gave statistically good fits for all the observations of the campaign and it provided very interesting information on each possible corona geometry. The disk-hot corona system agrees with a "photon-starved" configuration, i.e., a disk-corona geometry where the solid angle under which the corona "sees" the accretion disk is small. This is a common result for Seyfert galaxies since it is known that large covering 
factor coronae (e.g., slabs) cannot reproduce (due to the strong Compton cooling on the disk soft photons) hard X-ray spectra like those observed in Seyfert galaxies (e.g., Haardt 1993; Haardt et al. 1994). In contrast, the analysis of P13 shows that the warm corona agrees very well with a powerful, extended, and optically thick plasma covering a passive accretion disk, i.e., all the accretion power would be released in the warm corona. This is a result at odds with the commonly accepted behavior of standard optically thick, geometrically thin accretion flows (Shakura \& Sunyaev 1973) where the gravitational power is believed to be released in the deeper layers.

If true, the consequences are important, and have a direct impact on our understanding of the accretion disk vertical equilibrium, the expected spectral emission from such accretion flow, its capacity of producing outflows/jets, etc. Różańska et al. (2015) did a theoretical study of the existence of such a warm, optically thick corona at the surface of a standard accretion disk. By varying the accretion power released into the corona with respect to the underlying disk, as well as the magnetic pressure, this study shows that it is indeed possible to obtain solutions having the required temperature and optical depth (see their Fig. 4). Specifically, the best conditions are obtained when most of the power of accretion is released into the corona rather than the disk, in good agreement with the conclusion obtained with the Mkn 509 campaign. A high magnetic-to-gas pressure ratio is needed $(>30)$ to reach such large optical depth (10-20) while still ensuring hydrostatic balance ${ }^{1}$.

The purpose of the present paper is to test more consistently the warm corona model interpretation for the soft X-ray excess in radio quiet AGNs. We present in Sect. 2, the general equations governing the radiating equilibrium in a disk-corona structure. We show how these equations can be used to extract the physical properties of any corona (assuming its emission is dominated by thermal Comptonization) from the spectral fit parameters of its high-energy emission (mainly its photon index $\Gamma$ and cutoff temperature $k T$ ). Then, in Sect. 3, we compare these theoretical expectations with the different spectral analyses of the soft $\mathrm{X}$-ray excess from the literature and show their agreement with a quite extended corona geometry above a passive disk. However, none of these published spectral analyses consistently tests the two-coronae model on a large sample of AGNs. This is the goal of Sect. 4 where we describe our sample selection, our methodology, and our results before concluding.

\section{Disk-corona radiative equilibrium}

\subsection{Main equations}

In a disk-corona system, the Comptonizing region (i.e., the corona) and the source of soft photons (i.e., the disk) are coupled as the optically thick disk necessarily reprocesses and reemits part of the Comptonized flux as soft photons, which are the seeds for Comptonization. The system must then satisfy equilibrium energy balance equations, which depend mainly on the disk-corona geometry (see, e.g., Haardt \& Maraschi 1991; Stern et al. 1995). We develop a little in this section what these energy balance equations imply in terms of observables (see Appendix in Petrucci et al. 2013 for more details; see also Appendix B in Kubota \& Done 2016).

We assume that the accretion disk is mainly neutral, its temperature being rather low in AGNs. From the observed Comptonized spectrum, we can deduce an observed photon rate

\footnotetext{
1 The presence of outflows would relax this constraint, however.
}

$\dot{n}_{o b s}$. By conservation of the number of photons, which characterizes the Compton process ${ }^{2}$, this photon rate is equal to the sum of the seed photon rate crossing the corona without being Comptonized $\dot{n}_{s, 0}$ and those Comptonized in the corona and emitted upward (toward the observer) $\dot{n}_{c, u p}$. At first order,

$\dot{n}_{s, 0}=\dot{n}_{s} \mathrm{e}^{-\tau}$,

$\dot{n}_{c, u p}=\frac{\dot{n}_{s}\left(1-\mathrm{e}^{-\tau}\right)}{2}$,

where $\dot{n}_{s}$ is the photon rate emitted by the disk that enters the corona and the factor $1 / 2$ in Eq. (2) assumes the Compton scattering process to be isotropic with half of the Comptonized photons being emitted upward and the other half being emitted downward. The Compton process is certainly not isotropic, especially in the case of an anisotropic seed soft photon field (e.g., Haardt \& Maraschi 1991; Haardt 1993; Stern et al. 1995; Henri $\&$ Petrucci 1997), but the effect on the photon rate is relatively small.

In consequence, we have

$\dot{n}_{o b s}=\dot{n}_{s, 0}+\dot{n}_{c, u p}=\frac{\dot{n}_{s}\left(1+\mathrm{e}^{-\tau}\right)}{2}$,

which gives

$\dot{n}_{s}=2 \frac{\dot{n}_{o b s}}{\left(1+\mathrm{e}^{-\tau}\right)}$.

For an optically thin corona $(\tau \ll 1), \dot{n}_{s} \simeq \dot{n}_{o b s}$ (very few soft photons are Comptonized). On the other hand, for $\tau \gg 1$, $\dot{n}_{s} \simeq 2 \dot{n}_{o b s}$. Then, once the photon rate $\dot{n}_{s}$ of the accretion disk crossing the corona and the accretion disk temperature (deduced from the fit) are known, we have access to the luminosity $L_{s}$ coming from the disk that cools the corona.

On the other hand, the corona total power $L_{t o t}$ is the sum of $L_{S}$, the heating power $L_{h}$ liberated in the corona:

$L_{t o t}=L_{h}+L_{s}$.

Here, $L_{h}$ and $L_{s}$ can be divided into upward and downward:

$$
\begin{aligned}
L_{h} & =L_{h, u}+L_{h, d}=2 L_{h, u} \\
L_{s} & =L_{s, u}+L_{s, d} \\
& =\underbrace{L_{s} \mathrm{e}^{-\tau}+\frac{L_{s}\left(1-\mathrm{e}^{-\tau}\right)}{2}}_{L_{s, u}}+\underbrace{\frac{L_{s}\left(1-\mathrm{e}^{-\tau}\right)}{2}}_{L_{s, d}}
\end{aligned}
$$

The first equation assumes an isotropic Compton process, and the second equation is obtained with the same reasoning as in Eqs. (1) and (2).

Then, being $L_{r e f}$ the reflected luminosity at the disk surface, the observed luminosity can be written

$L_{o b s}=L_{h, u}+L_{s, u}+L_{r e f}$.

$L_{r e f}$ is directly related to the down-scattered corona emission

$$
\begin{aligned}
L_{r e f} & =a\left(L_{s, d}+L_{h, d}\right) \\
& =a\left(\frac{L_{s}\left(1-\mathrm{e}^{-\tau}\right)}{2}+\frac{L_{h}}{2}\right),
\end{aligned}
$$

\footnotetext{
2 We neglect the pair creation/annihilation process here, which is a reasonable assumption for low-temperature plasma $(<500 \mathrm{keV})$. We also neglect the photon rate from the reflection component. For a neutral accretion disk, it is negligible in comparison to the disk photon rate.
} 
P.-O. Petrucci et al.: Testing warm Comptonization models for the origin of the soft X-ray excess in AGNs

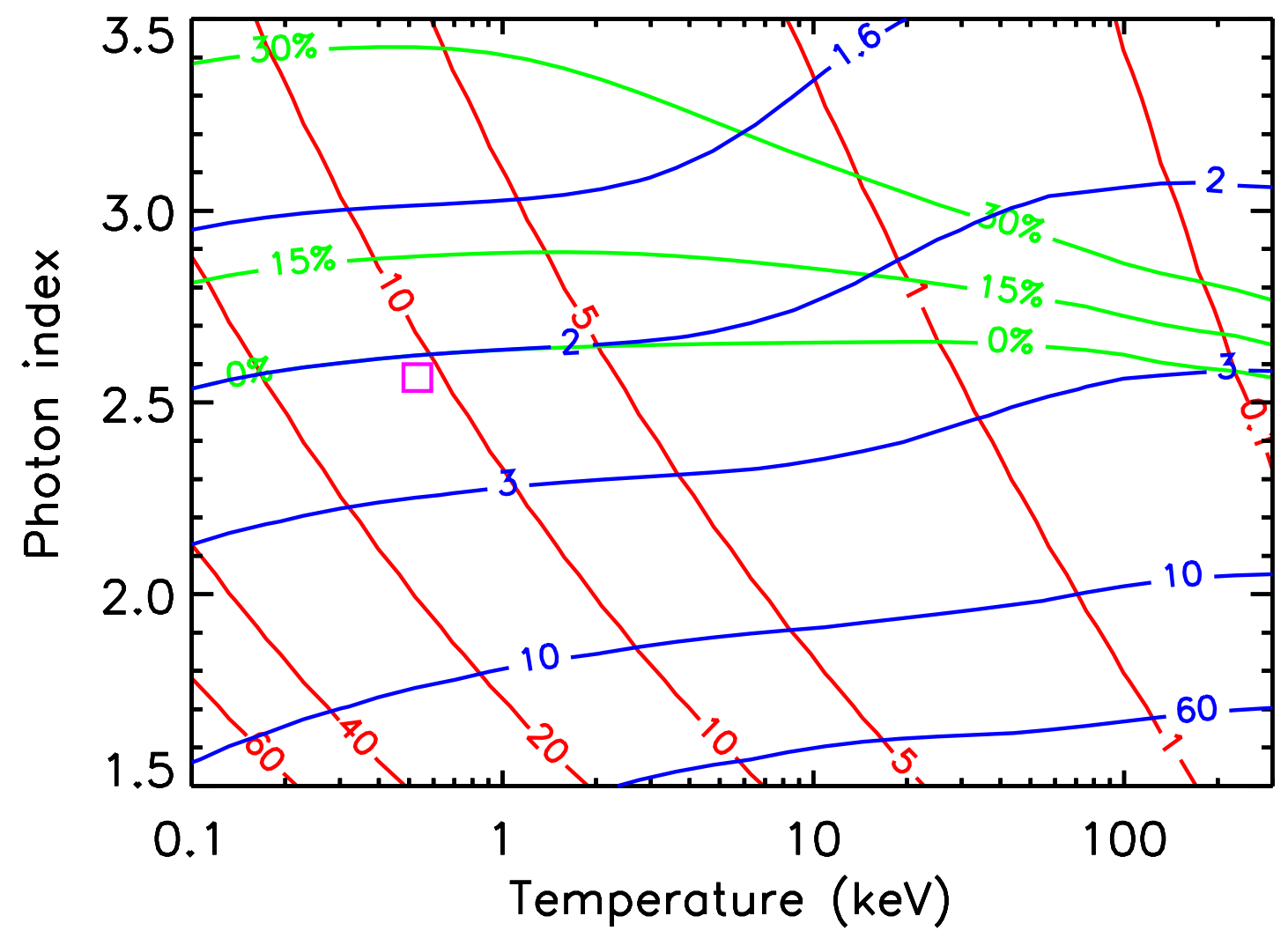

Fig. 1. Contours of the corona optical depth $\tau$ (in red), the amplification factor $A$ (in blue) and the minimal fraction (in \%) of disk intrinsic emission $\left.\frac{L_{d i s k, i n t r}}{L_{S}}\right|_{\text {min }}$ (in green) in the corona spectral parameter $\Gamma-k T$ plane. A slab geometry (i.e., $g=1$ in Eq. (21)) is assumed here. Smaller values of $g$ (i.e., a patchy corona) would move the green contours down. The magenta rectangle corresponds to the $\Gamma$ and temperature $k T_{e}$ ranges obtained by P13 for the soft excess in the spectral analysis of the Mkn 509 campaign.

where $a$ is the disk albedo. Since we assume the disk to be neutral, the albedo is not expected to be large. As shown by Haardt \& Maraschi (1993, see their Fig. 2), in a radiatively coupled diskcorona system the albedo depends on the corona optical depth and is on the order of $10 \%$ for small corona optical depth $\tau \sim 0.1$. It rapidly decreases to 0 as $\tau$ increases since the corona spectrum softens and is efficiently absorbed by the neutral disk matter. We take into account the albedo dependency with $\tau$ by digitalizing and interpolating the function $a(\tau)$ plotted in Fig. 2 of Haardt \& Maraschi (1993) and assuming $a=0$ for $\tau>1$.

From Eqs. (6), (8), and (9), we can deduce $L_{h}$

$L_{h}=2 L_{o b s}-L_{s}\left(1+\mathrm{e}^{-\tau}\right)-2 L_{r e f}$,

and finally, from Eq. (5)

$L_{t o t}=2 L_{o b s}-L_{s} \mathrm{e}^{-\tau}-2 L_{r e f}$.

Let us introduce the Comptonization amplification factor $A$ defined by $L_{t o t}=A L_{s}$. From the above equations, we deduce that

$A \simeq 2 L_{\text {obs }} / L_{s}-2 L_{r e f} / L_{s}-1$, for $\tau \ll 1$

$A \simeq 2 L_{o b s} / L_{s}-2 L_{r e f} / L_{s}$, for $\tau \gg 1$.

The radiative equilibrium of the disk also implies

$L_{\text {disk }}=(1-a)\left(L_{s, d}+L_{h, d}\right)+L_{d i s k, i n t r}$,

where $(1-a)\left(L_{s, d}+L_{h, d}\right)$ is the corona emission reprocessed in the disk and $L_{\text {disk,intr }}$ is the intrinsic disk emission (i.e., locally generated in the disk). Concerning the luminosity $L_{s}$, it characterizes the part of the disk emission $L_{\text {disk }}$ entering and cooling the corona. It is equal to $L_{d i s k}$ if the corona entirely covers the accretion disk, but it is lower than $L_{\text {disk }}$ in the case of a patchy corona where part of the disk emission reaches the observer without crossing the corona. So we have

$$
\begin{aligned}
L_{s} & <(1-a)\left(L_{s, d}+L_{h, d}\right)+L_{d i s k, i n t r} \\
& =g\left[(1-a)\left(\frac{L_{s}\left(1-\mathrm{e}^{-\tau}\right)}{2}+\frac{L_{h}}{2}\right)+L_{\text {disk,intr }}\right],
\end{aligned}
$$

where $g$ is a geometrical parameter, $\leq 1$, related to the patchiness of the corona. Geometrically, it gives an estimate of the solid angle $\Omega$ under which the disk sees the corona, i.e., $g=\Omega / 2 \pi$. In consequence, the heating-to-cooling ratio is equal to

$\frac{L_{h}}{L_{s}}=A-1=\frac{2}{1-a}\left(\frac{1}{g}-\frac{L_{d i s k, i n t r}}{L_{s}}\right)+\left(\mathrm{e}^{-\tau}-1\right)$.

which gives, thanks to Eqs. (11) and (12),

$\frac{L_{d i s k, \text { intr }}}{L_{s}}=\frac{1}{g}+\frac{2(1-a)}{2+a}\left(\mathrm{e}^{-\tau}-\frac{L_{o b s}}{L_{s}}\right)$
$\frac{L_{d i s k, \text { intr }}}{L_{s}}=\geq 1+\frac{2(1-a)}{2+a}\left(\mathrm{e}^{-\tau}-\frac{L_{o b s}}{L_{s}}\right)=\left.\frac{L_{d i s k, \text { intr }}}{L_{s}}\right|_{\text {min }}$.

In the case of an optically thin $(\tau \ll 1)$ corona entirely covering $(g=1)$ a passive $\left(L_{\text {disk,intr }}=0\right)$ and nonreflective $(a=0)$ disk, we found the well-known result $L_{h} / L_{s}=2$ (see, e.g., 
Haardt \& Maraschi 1991 and their Eq. (3b) with $f=0$ and no disk albedo) and consequently the amplification ratio $A=$ $L_{t o t} / L_{s}=3$. On the other hand, for an optically thick corona (still entirely covering a passive disk with no albedo), we find $L_{h} / L_{s}=1$ and $A=L_{t o t} / L_{s}=2$. Higher values of $A$ compared to these fiducial values necessarily required $g<1$, i.e., a patchy corona. On the other hand, lower values of $A$ imply $L_{\text {disk,intr }}>0$.

\subsection{Mapping}

For a given geometry, Eqs. (12), (13), and (21) link the model parameters $\left(L_{o b s}\right.$ and $\left.\tau\right)$ to the intrinsic characteristics $\left(A=L_{t o t} / L_{s}\right.$ and $\left.\left.\frac{L_{d i s k, i n t r}}{L_{s}}\right|_{\min }\right)$ of the corona-disk system. It is then possible to "map" one pair of parameters in the 2D plane of the other pair. To this end, we used the Comptonization model NTHCOMP (Zdziarski et al. 1996; Życki et al. 1999) in XSPEC (Arnaud 1996; Arnaud et al. 1999). NTHCOMP is characterized by three main parameters: the corona electron temperature $k T_{e}$, the disk photon temperature $k T_{b b}$ (we assume a multicolor disk blackbody), and the power-law photon index $\Gamma$ of the Comptonized spectrum. Then we proceed through the following steps:

- We first assume a disk photon temperature $k T_{b b}$. Let us assume $3 \mathrm{eV}$, but the result is weakly dependent on this parameter as long as it is on the order of a few eVs.

- We choose a set of model parameter values $\Gamma, k T_{e}$, and produce a SED.

- From $\Gamma$ and $k T_{e}$ we estimate the corresponding optical depth $\tau$ using Eq. (13) in Beloborodov $(1999)^{3}$, i.e., $\Gamma \simeq \frac{9}{4} y^{-2 / 9}$ with $y=4\left[k T_{e} / m_{e} c^{2}+4\left(k T_{e} / m_{e} c^{2}\right)\right] \tau(\tau+1)$ the so-called Compton parameter. From the SED integration, we also compute $L_{o b s}$ and $\dot{n}_{o b s}{ }^{4}$.

- Then we estimate $L_{s}$ for the given $T_{b b}$ by assuming photon conservation in slab geometry using Eq. (4).

Following this procedure, and for a given $k T_{b b}$, we can compute $\tau, L_{o b s}$, and $L_{s}$; similarly, given Eqs. (14), (15), and (21), we can compute $\tau, A$, and $\left.\frac{L_{d i s k, i n t r}}{L_{s}}\right|_{\min }$ in function of $\Gamma$ and $k T_{e}$. We show in Fig. 1 the corresponding contours of the optical depth $\tau$ (in red), the amplification factor $A$ (in blue), and the minimal fraction of disk intrinsic emission $\left.\frac{L_{\text {disk,intr }}}{L_{s}}\right|_{\min }$ (in green) in the $\left(\Gamma, k T_{e}\right.$ ) plane for $\Gamma$ varying between 1.5 and 3.5 and $k T_{e}$ between $0.1 \mathrm{keV}$ and $300 \mathrm{keV}$.

The contour $\left.\frac{L_{\text {disk,intr }}}{L_{s}}\right|_{\text {min }}=0 \%$ corresponds to the theoretical case of a slab corona above a passive disk. This contour divides Fig. 1 into two parts. Above it, a slab corona-disk geometry can exist: the farther up, the larger the disk intrinsic emission. On the other hand, below it the disk-corona geometry is necessarily patchy. We also note that at large optical depth (left part of the figure), this contour agrees with an amplification factor of 2 , while at low optical depth (right part of the figure) it agrees with $A=3$, as theoretically expected.

Since observations provide us with $\Gamma$ and $k T_{e}$, we are able to deduce, for a given object, the corresponding values of $\tau, A$, and $\left.\frac{L_{\text {disk,intr }}}{L_{s}}\right|_{\min }$.

\footnotetext{
3 This is an approximative estimate of $\tau$, but it is sufficient for our current purposes.

4 The units of $L_{o b s}$ and $\dot{n}_{o b s}$ do not matter here because we are dealing with luminosity and photon rates ratios.
}

Interestingly, the contours $\left.\frac{L_{\text {disk,intr }}}{L_{s}}\right|_{\min }=0 \%$ and $A=2$ correspond, for large optical depths, to corona photon index $\Gamma \sim$ 2.5-2.6. This is indeed the photon index value for which $\dot{n}_{s} \simeq$ $2 \dot{n}_{o b s}$ and $L_{s} \simeq L_{o b s}$ (Eqs. (4) and (15), respectively, with $\tau \gg 1$ ) are satisfied (see details in Appendix A). This also corresponds to the situation $L_{h}=L_{s}$, i.e., a corona heating on the order of the disk luminosity.

\section{Indication of a dominantly dissipating and extended warm corona for the origin of the soft $\mathrm{X}$-ray excess}

As said in the introduction, the two-coronae model was carefully tested with the optical/UV/X-ray data set from the broadband campaign on Mrk 509 (P13). This spectral analysis showed that the warm corona spectral emission was quite well constrained with a photon index $\Gamma$ and temperature $k T_{e}$ varying in the ranges 2.53-2.6 and 0.48-0.59 keV, respectively. Looking at Fig. 1, and conformably to the conclusions of P13, these parameter values agree with an optically thick corona ( $\tau$ between 10 and 20) in a slab geometry $(A \simeq 2)$ above a passive $\operatorname{disk}\left(\left.\frac{L_{\text {disk,intr }}}{L_{s}}\right|_{\text {min }} \simeq 0\right)$.

Interestingly, past spectral analyses of the soft X-ray excess on large samples of objects show similar values for $\Gamma$ and, when included in the modeling, of $k T_{e}$. From their analysis of the $0.1-$ $2.4 \mathrm{keV}$ ROSAT spectra of 58 Seyfert 1, Walter \& Fink (1993) found photon indices varying between 2.0 and 3.2 with a mean of 2.5 (see their Fig. 2). Similar results for $\Gamma$ were obtained by Brunner et al. (1997), Laor et al. (1997), and Schartel et al. (1996) from their analysis of the ROSAT spectra of different quasar samples. More recently, Brocksopp et al. (2006) analyzed XMMNewton EPIC-pn and OM observations for 22 Palomar Green quasars. They obtained a reasonable fit of the $0.3-10.0 \mathrm{keV}$ spectra of most of the sources with a broken power law (plus an iron line), the low-energy power-law (to fit the soft excess) photon index falling in the 2.0-4.0 range, with an average value around 2.9 .

A few years ago, Jin et al. (2012b) presented the broadband (from optical to hard X-rays) spectral analysis of a sample of 51 unobscured Type 1 AGNs, fitting together XMM-Newton and Sloan Digital Sky Survey spectra with a model very similar to the two-coronae model. It assumes that the gravitational potential energy is emitted as optically thick blackbody emission at each radius down to some specific coronal radius. Below this radius the remaining energy down to the last stable orbit is divided between two coronae that play the same roles of the warm and hot coronae of the two-coronae model (the slab geometry is also discussed in the Appendix of Done et al. 2012). The main difference with our approach is that their model assumes, by construction, intrinsic disk emission. Their spectral analysis shows that the warm corona temperatures and optical depths ${ }^{5}$ of the whole sample cluster are around $0.2-0.3 \mathrm{keV}$ and $10-20$, respectively. While these parameter values theoretically agree with their assumptions of a patchy warm corona above an intrinsically radiative disk (indeed for $g<1$ the green contours of $\left.\frac{L_{\text {disk,intr }}}{L_{s}}\right|_{\min }$ move down in Fig. 1), it is quite surprising that they also agree very well with a slab corona above a passive disk.

\footnotetext{
5 Their Comptonization model for the warm corona is COMPTT in XSPEC. COMPTT uses temperature and optical depth as the corona parameters.
} 
These studies show that, whatever the model used for the soft $\mathrm{X}$-ray excess, the data constrain the spectral parameters to fall in the region of the $\Gamma-k T$ space that is consistent with a dominantly dissipating and extended warm corona, in good agreement with the conclusions of P13 for Mkn 509. Most of the past works used only a phenomenological power-law model to fit the soft $\mathrm{X}$-ray excess, but did not include the optical/UV data. On the other hand, the detailed spectral analysis of Jin et al. (2012b) includes the optical/UV and soft X-ray data and use realistic Comptonization modeling, but their model assumes intrinsically that a significant part of the optical/UV is directly produced by the accretion disk.

The goal of the following section is to test the results obtained on Mkn 509 on a larger sample of radio quiet AGNs by fitting optical/UV, soft X-ray up to hard X-rays data $(10 \mathrm{keV})$ with the two-coronae model. Contrary to past analyses, in the two-coronae model, the optical/UV to soft X-ray emission is entirely due to the warm corona. We first want to check if this model can fit other AGNs reasonably well and, second, to see how the warm corona best-fit parameters compare with the theoretical expectations discussed in Sect. 2.

\section{Testing the two-coronae model on a sample of AGNs}

\subsection{Sample selection and data reduction}

To test the two-coronae model, we need bright sources with simultaneous data in the optical/UV and X-ray bands. Simultaneity is important here because these sources are generally variable at all wavelengths on timescales of day or weeks (e.g., Ponti et al. 2012). The capabilities of XMM-Newton are optimal for this task, as it provides both high-quality X-ray spectra with the EPIC-pn camera (Strüder et al. 2001) and optical/UV data with the optical monitor (OM; Mason et al. 2001). Our sample was thus built starting from the sources observed by XMM-Newton, with public data as of April 2014, cross-correlated with the AGN and quasar catalog of Véron-Cetty \& Véron (2010). The sources were further selected using the criteria of the CAIXA catalog (Bianchi et al. 2009), i.e., the sources are radio-quiet and unobscured $\left(N_{H}<2 \times 10^{22} \mathrm{~cm}^{-2}\right)^{6}$.

In addition to this, and in order to have better constraints in the optical/UV range during the fitting procedure, we want the largest number of $\mathrm{OM}$ filters for each source of the sample. Indeed, the variability (in flux and shape) of the sources in the OM band is expected to be small (e.g., Gelbord et al. 2015) so that the different OM filter measurements could be used simultaneously to give reasonable constraints to the disk emission (see Sect. 4.2.2 for more details). For this purpose, we first cross-correlate the initial sample with the XMM-Newton/OM serendipitous UV source survey catalog (OMSUSS v2.1, Page et al. 2012). We then select the largest number of observations per source in order to have at least four OM filter detections. The complete sample of sources and corresponding ObsIDs is listed in Table B.1. It corresponds to 22 objects and 100 ObsIDs.

The data reduction is identical to that detailed in Bianchi et al. (2009); i.e., the EPIC-pn data were all reprocessed with the most updated versions of the SAS software (Gabriel et al. 2004). For the observations performed in Small Window mode,

\footnotetext{
6 At this selection step, sources like $1 \mathrm{H}$ 0707-495 and Mrk 766 have been discarded from the sample owing to their known complex absorption/reflection components (e.g., Gallo et al. 2004; Sako et al. 2003).
}

background spectra were generated using blank-field event lists, following the procedure presented in Read \& Ponman (2003). In all other cases, background spectra were extracted from sourcefree regions close to the target in the observation event file. Source extraction radii and screening for intervals of flaring particle background were performed via an iterative process that leads to a maximization of the signal-to-noise ratio, similar to that described in Piconcelli et al. (2004).

Spectra were binned in order to oversample the intrinsic instrumental energy resolution by a factor not lower than 3 and to have spectral bins with at least 25 background-subtracted counts. This ensures the applicability of the $\chi^{2}$ statistics.

\subsection{Methodology}

\subsubsection{The model components}

In this section, we describe the different model components used to fit our sample of optical/UV-X-ray spectra. Since we were mostly interested in the continuum spectral parameters, we did not need a very precise description of the data and we made the choice of fitting with a limited number of physically motivated spectral components. The main assumption of the two-coronae model is that the optical/UV emission is the signature of an optically thick multicolor accretion disk whose optical/UV photons are Comptonized in two different media: the first (the so-called warm corona) produces the soft X-ray excess, and the second (the so-called hot corona) the high-energy continuum emission. The different model components described below are also listed in Table 1. We also indicate in this table the parameters of each component that are left free to vary (or not) during the fitting procedure.

Continuum. We used the NTHCOMP model of XSPEC to model each of the corona Comptonization emissions. As said before, the free parameters of NTHCOMP are the electron temperature of the corona $k T_{e}$, the soft-photon temperature $k T_{b b}$, and the asymptotic power-law index $\Gamma$. In the following, we will use the subscripts "wc" for the warm corona parameters and "hc" for the hot corona. Due to the lack of data above $10 \mathrm{keV}$, the temperature of the hot corona is fixed to $100 \mathrm{keV}$. We also assume the same soft-photon temperature for the two coronae.

Reflection component. We include a reflection component (the XILLVER model in XSPEC, García \& Kallman 2010) leaving only the normalization as a free parameter. Indeed, the main feature of XILLVER in the $0.3-10 \mathrm{keV}$ band is the $\mathrm{Fe} \mathrm{K}_{\alpha}$ line, plus a contribution to the continuum above $8 \mathrm{keV}$. Therefore, the spectral shape of the illuminating radiation of XILLVER is not crucial. We thus fixed the photon index of XILLVER to the arbitrary value of 1.9 and assumed an iron abundance of 1 and an ionization parameter $\log \xi=0$. We checked that linking the photon index of XILLVER to that of the primary continuum does not alter the results much. We also fixed the power-law cutoff energy to $E_{c}=3 k T_{e}=300 \mathrm{keV}$ (Petrucci et al. 2001).

Neutral and warm absorption. Concerning the presence of absorption, we first assume a Galactic neutral hydrogen column density $N_{h}$ (TBABS model of XSPEC), appropriate for the sky coordinates of the source, from Kalberla et al. (2005). The value of $N_{h}$ is fixed during the fitting procedure. We add a warm absorber (WA) component modeled with a CLOUDY table, leaving free the column density and the ionization parameter ("cloudytable" model component in Table 1; see Cappi et al. 2016 for more details on this CLOUDY table). 
Reddening, small blue bump, and Galactic emission. Since we use optical/UV data, we must take into account two main components that can give a significant contribution in the optical/UV band. The first is the host galaxy ("galaxy" model component in Table 1), especially in the optical band, its UV emission being expected to be negligible. The second main component is the broad-line region, which is responsible for the so-called small blue bump around $3000 \AA$ ("smallBB" model component in Table 1). We refer the reader to Mehdipour et al. (2015) for a detailed description of these contributions in the case of the NGC 5548 XMM-NuSTAR campaign (Kaastra et al. 2014). For the galaxy contribution, we assumed the same template spectrum as the one used by Mehdipour et al. (2015) for NGC 5548, the precise spectral shape being of weak importance when using only six broadband filters. Finally, we included the reddening (REDDEN model in XSPEC), calculated from the Galactic extinction following Güver \& Özel (2009). The reddening is fixed during the fitting procedure.

The complete model we used, in XSPEC terms, is then

$$
\begin{aligned}
& \text { tbabs } \times \text { redden } \times \text { mtable }\{\text { cloudytable }\} \times\left(\text { nthcom } p_{w c}\right. \\
& \quad+\text { nthcom } p_{h c}+\text { atable }\{\text { smallBB }\}+\text { atable }\{\text { galaxy }\} \\
& \quad+\text { xillver })
\end{aligned}
$$

This model has nine free parameters (see Table 1). For each source, the redshift is taken from the NASA/IPAC Extragalactic Database (NED) or from the SIMBAD database operated at CDS, Strasbourg.

In several cases, however, our best fits show residuals of emission/absorption line-like features below $1 \mathrm{keV}$ and around $2 \mathrm{keV}$. Even though they are rather weak (typically a few eV equivalent width), they can be statistically significant depending on the statistics of the data. Part of the residuals are due to calibration issues (see Cappi et al. 2016 for a detailed discussion). Those around $2 \mathrm{keV}$ are probably ascribed to remaining systematic calibration uncertainties owing to the detector quantum efficiency at the Si K-edge $(1.84 \mathrm{keV})$ and mirror effective area at the Au M-edge $(\sim 2.3 \mathrm{keV})$. It was then decided to cut the $1.8-2.4 \mathrm{keV}$ part of the spectrum out. Features at energies lower than $\sim 1.5 \mathrm{keV}$ could be modeled by a combination of a few narrow absorption and/or emission lines at energies around $\sim 0.5-0.6 \mathrm{keV}$ and $1-1.1 \mathrm{keV}$, and $\mathrm{EW}$ variable between $\sim 8-15 \mathrm{eV}$, depending on the line and observation considered. We estimate that the origin of these features could be ascribed to either remaining uncertainties in the CTI energy scale at low energies in the pn data, or to an improper (or approximate) modeling of the emission and absorption lines.

Another part of the residuals can also be due to bad modeling of the WA features. The goal here, however, is not to obtain a precise fit of the WA. We are interested in the continuum and we believe that our results are not significantly impacted by the WA modeling. We discuss this point in more detail in Sect. 4.3.1. This is also supported by the fact that our methodology applied to Mkn 509 gives results in good agreement with those obtained by P13 where the WA was precisely taken into account. In some cases, however, and if the improvement is indeed significant, we added another WA component (see next section).

It is important to point out that no link between the Comptonized spectrum and the soft UV emission is imposed a priori in our modeling. The two-coronae model simply adjusts its parameters, independently of each other, to fit the data. It is only a posteriori that the resulting best fit values of the coronae characteristics can be interpreted in a physically motivated scenario.
Table 1. Model components and parameters of the two-coronae model described in Sect. 4.2.1.

\begin{tabular}{ccc}
\hline \hline $\begin{array}{c}\text { Model component } \\
\text { in XSPEC }\end{array}$ & Free parameters & Fixed parameters \\
\hline tbabs & - & $N_{h}$ \\
redden & - & $E(B-V)$ \\
cloudytable & $N_{h}, \xi$ & $v_{t u r b}=100$ \\
smallBB & - & norm \\
galaxy & - & norm \\
nthcomp & - & - \\
& $\Gamma_{w c}, T_{e, w c}$ & $T_{e, h c}=100 \mathrm{keV}$ \\
nthcomp $_{h c}$ & $T_{b b, w c}$, norm $_{w c}$ & $T_{b b, h c}=T_{b b, w c}$ \\
& & $\Gamma=1.9, \xi=1$ \\
xillver & norm & $E_{c}=300 \mathrm{keV}$ \\
& & $i=30^{\circ}, A_{F e}=1$ \\
\hline
\end{tabular}

Notes. ${ }^{(1)}$ The normalizations of the "smallBB" and the "galaxy" templates are fixed to their best fit values obtained when fitting the OM data only (see Sect. 4.2.2 for more details on the fitting procedure).

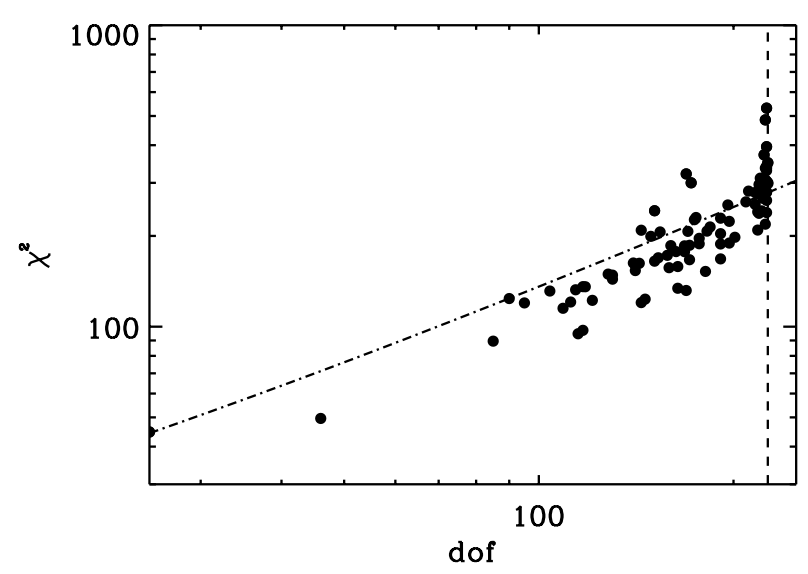

Fig. 2. Distribution of our best fit $\chi^{2}$ in function of the degree of freedom (dof) of each observation of our sample. The dot-dashed curve indicates the $\chi^{2}$ value corresponding to a $99 \%$ null hypothesis probability for the corresponding dof value. The vertical dashed line represents the 226 dof limit of our sample.

\subsubsection{Fitting procedure}

Given the large number of observation data sets, we developed an automatic fitting procedure that we describe below. For each object, the main steps are as follows:

1. Fitting all the OM filters of all the ObsIDs of the same object with a model composed of a disk blackbody (DISKBB in XSPEC), the smallBB, and the galaxy templates. The temperature of the disk, the smallBB, and galaxy normalizations are left free to vary, but are tied between the different ObsID, while the disk normalizations are left free to vary between all the ObsIDs. We also include the reddening estimated as explained in the previous section. From the resulting best fit, we extract the normalizations of the smallBB and galaxy, as well as the disk temperature.

2. Fitting the $\mathrm{OM}+\mathrm{PN}$ data of all the ObsIDs of the same object with the two-coronae model, fixing the disk temperature in the NTHCOMP models and the normalizations of the smallBB and galaxy components to the values obtained in 


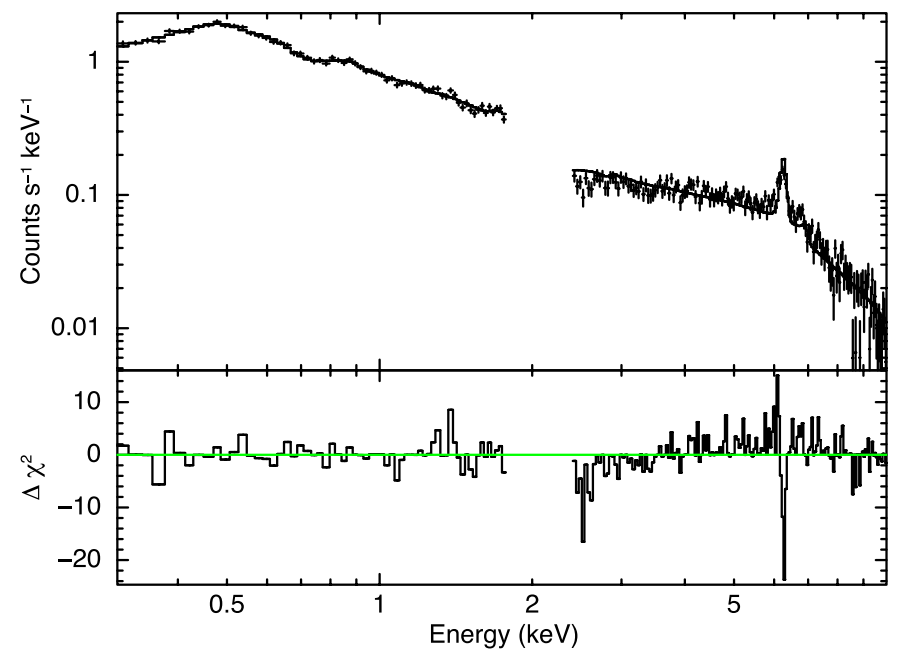

Fig. 3. "Best fit" for UGC 3973/ObsID:0502091001, actually the worst fit obtained with our automatic fitting procedure. The presence of a feature close to the iron line is clearly visible.

the preceding step. A first fit is done and then we leave the disk temperature free to vary and refit the data again.

This procedure provides us with the two-coronae model best fits of all the ObsIDs of a given object. While we obtain statistically acceptable best fits for a significant number of observations, a part of the sample gives quite poor results, the script converging to unrealistic parameter values, e.g., too small $\Gamma_{h c}$, on the order of unity, or too high disk temperature, $T_{b b}>0.1 \mathrm{keV}$. A one-by-one analysis was then done. The too high disk temperature generally occurred when the optical/UV data of the OM filters did not constrain $T_{b b}$ well and we needed to fix it to its best fit value obtained in step 1 to successfully fit the $\mathrm{OM}+\mathrm{PN}$ data together. Strong residuals of emission/absorption line-like features may also be present below $1 \mathrm{keV}$, and we add two Gaussian lines as explained in Sect. 4.2.1. In a very limited number of cases, we also add another WA component (still using a CLOUDY table). This is generally the case for objects with known complex warm absorption (see Appendix D for a discussion of these particular model fitting procedures).

\subsection{Results}

\subsubsection{Goodness of fits}

The final distribution of the $\chi^{2}$ values we obtained as a function of the degrees of freedom (dof) of each observation is shown in Fig. 2. The region where the adopted model can be considered correct at the $99 \%$ confidence level is delimited by the dot-dashed curve which corresponds, for each dof value, to a $1 \%$ probability of getting a value of $\chi^{2}$ as high as or higher than the value we found if the model is correct. Two thirds of our fits $(60 \%)$ lie under this curve, confirming the reasonable quality of the derived spectral parameter $(90 \%$ of our sample has $\left.\chi^{2} / \operatorname{dof}<1.5\right)$.

Not surprisingly, most of the observations of the remaining one-third have very large dof values due to their high $\mathrm{S} / \mathrm{N}$ spectra.

There are generally two reasons for the poor fit quality: either a poor fit of the iron line complex and/or the presence of strong residuals in the soft X-ray part of the spectrum, where the effects of absorption from ionized matter are only roughly taken into account by our modeling. We have checked that the addition of further spectral components (WA, absorption/emission lines as explained in Sect. 4.2) indeed improves the fit without modifying significantly the spectral properties of the continuum. As an example, we show in Fig. 3 the best fit data for UGC 3973 $($ ObsID $=0502091001)$ whose reduced $\chi^{2}(530 / 225)$ is among the worst in the sample. While the other data sets of UGC 3973 give acceptable fits (see Table C.1 in Appendix C), this ObsID shows a clear absorption feature close to the $\mathrm{FeK} \alpha$ line. The addition of a narrow $(\sigma=0 \mathrm{keV})$ Gaussian line in absorption drastically improves the fit $\left(\Delta \chi^{2}=160\right.$ for two dof less) with a Gaussian best fit energy $E_{\text {gau }}=6.40 \pm 0.01 \mathrm{keV}$ (rest frame) and $\mathrm{EW}=180 \pm 30 \mathrm{eV}$. After the addition of this absorption line, the best fit parameter values for the warm and hot corona become $\Gamma_{w c}=2.44 \pm 0.03, k T_{w c}=0.23 \pm 0.15 \mathrm{keV}$, and $\Gamma_{h c}<1.65$. This absorption feature suggests a bad fit of the iron line profile. So we also test a more physical model by blurring (KDBLUR model in XSPEC) the reflection component given by XILLVER. The inner disk radius and the inclination of KDBLUR are left free to vary in the fit. The new best fit is better $\left(\Delta \chi^{2}=103\right.$ for two dof less $\left.{ }^{7}\right)$, to a lesser extent however, compared to the absorption Gaussian line. This could indicate an even more complex iron line profile whose precise modeling is beyond the scope of the present analysis. The best fit parameter values for the warm and hot corona become $\Gamma_{w c}=2.41 \pm 0.03, k T_{w c}=0.20 \pm 0.01 \mathrm{keV}$, and $\Gamma_{h c}<1.55$. These values should be compared to those obtained with the automatic procedure, i.e., $\Gamma_{w c}=2.40 \pm 0.03, k T_{w c}=$ $0.19 \pm 0.01 \mathrm{keV}$, and $\Gamma_{h c}=1.5_{-0}^{+0.14}$. Since the present analysis is focusing on the continuum, we safely conclude that, even when the statistical significance of the best fit is low, the values of the $\mathrm{X}$-ray parameters derived from the fit are still very reliable and can be used to properly characterize the source continuum.

This is the first important result of the application of the two-coronae model to a large sample of optical/UV/X-rays AGN spectra. The two-coronae model assumes that the optical/UV to soft X-ray emission is entirely explained by a unique warm corona component. In addition, this model fully agrees, statistically, with the optical/UV/X-rays spectra of several AGNs without the need of an additional disk component in the optical/UV band.

We give three examples among our best fits in Fig. 4. At top of each figure, we show the OM and PN data with the unfolded best fit model, and the corresponding data/model ratios at the bottom. These examples correspond to observations of three different objects with different warm corona photon indexes covering the range of $\Gamma_{w c}$ obtained in our sample, i.e., 2.24, 2.68, and 2.99 for PG1114+445, LBPQS1228+1116, and PG1116+215, respectively.

\subsubsection{Physical properties of warm corona}

The best fit parameter values of the warm corona (i.e., photon index $\Gamma_{w c}$ and temperature $k T_{e, w c}$ ) and of the hot corona (i.e., photon index $\Gamma_{h c}$, the reduced $\chi^{2}$ and the degrees of freedom) are reported in Table C.1 of Appendix C. The warm corona photon index and temperature are also reported in Fig. 5 for all the sources of our sample and the histograms of their distribution are shown in Fig. 6. The optical depth contour plots (from Fig. 1) are overplotted in Fig. 5 to ease the comparison.

The $\Gamma_{w c}$ values are distributed between 2 and 3, with a clear preferential value around 2.6-2.7. The warm corona temperature $k T_{e, w c}$ is in the $0.1-1 \mathrm{keV}$ range, but it is preferentially smaller

\footnotetext{
7 The best fit parameter values of KDBLURR are $R_{\text {in }}=100_{-40}^{+110}$ and $i=19_{-6}^{+3}$.
} 

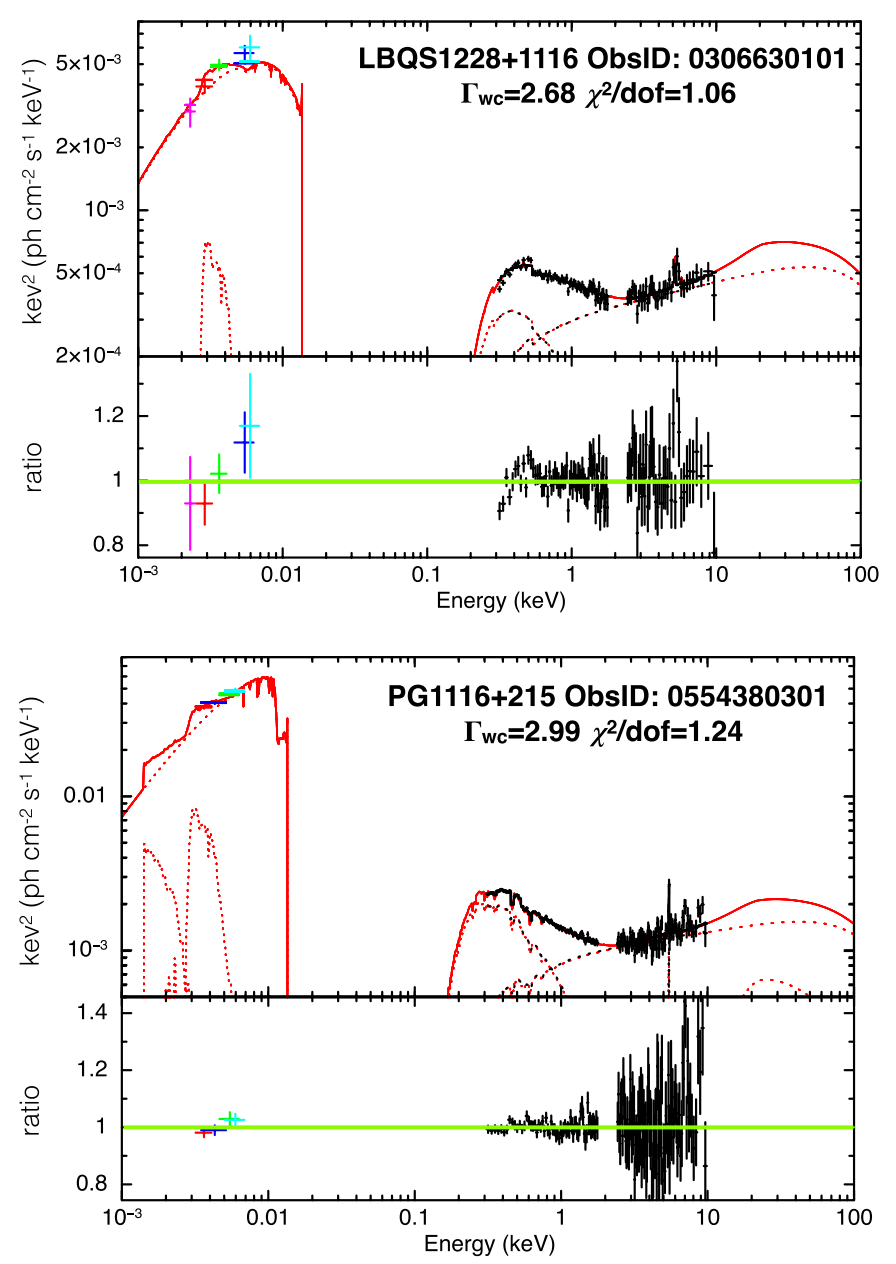

than $0.4 \mathrm{keV}$. This corresponds to a region of the $\Gamma-k T$ plane with large optical depths; most of the sources agree with $\tau_{\text {warm }}>10$. This is in agreement with similar spectral analysis published in the literature (e.g., Petrucci et al. 2013; Jin et al. 2012b). The dashed blue histograms in Fig. 6 correspond to the histograms of the averaged values of $\Gamma_{w c}$ and $k T_{e, w c}$ for each object in our sample. They show roughly the same trends as those observed for the whole observation sample.

For comparison, the best fit photon indexes of the hot coronae $\Gamma_{h c}$ are overplotted in Fig. 5 (black circles on the right). The temperature of the hot corona being fixed to $100 \mathrm{keV}$ in the fits, they are all aligned vertically. The histogram of the $\Gamma_{h c}$ values is overplotted in Fig. 6. It peaks between 1.5 and 2. Also shown in Fig. 5 (gray filled circles) are the best fit parameters of the hot coronae from Fabian et al. (2015). The warm and hot coronae parameters a clearly separated, the hot coronae parameters clustering at the bottom right part of the figure with harder spectra $(\Gamma<1.9)$ and higher temperature $(k T>20 \mathrm{keV})$ characteristic of the highenergy emission of Type 1 objects. The hot corona optical depths are also on the order of unity as generally observed (e.g., Petrucci et al. 2001; Matt et al. 2014; Brenneman et al. 2014a,b).

\subsubsection{A slab-like geometry above a passive accretion disk}

We show our best fit parameter values for the warm corona in Figs. 7 and 8 where the contours (from Fig. 1) of the amplification factor and $\left.\frac{L_{\text {disk,intr }}}{L_{s}}\right|_{\text {min }}$, respectively, are overplotted. The warm corona amplification ratios are distributed between

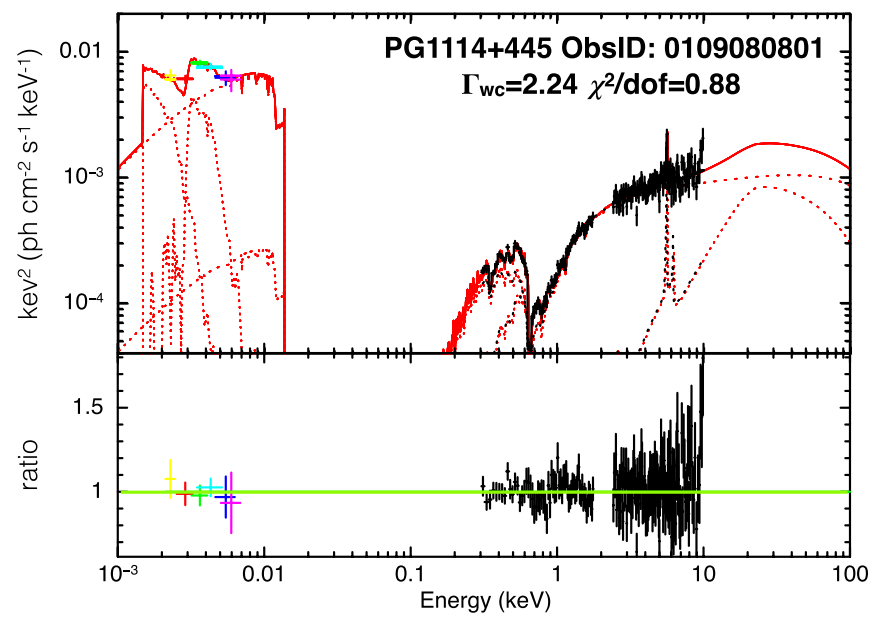

Fig. 4. Examples of best fit SED and the data/model ratio for three observations with different warm corona photon indexes covering the range observed in our sample. The source names, ObsID, $\Gamma_{w c}$, and reduced $\chi^{2}$ are indicated in each plot. The black crosses represent the $\mathrm{XMM} / \mathrm{PN}$ data and the colored crosses the different XMM/OM filters. The red solid line is the absorbed best fit model, while the dotted lines represent the different model components: hot and warm corona emission, reflection component, host galaxy, and small blue bump emission.

1.6 and 3, with a peak (corresponding to the peak of the photon index around 2.6-2.7 in Fig. 6) close to 2 . This is precisely the theoretical value of a thick corona in slab geometry and in radiative equilibrium with a passive disk, as discussed in Sect. 2.1.

This is also shown in Fig. 8, since all the objects agree with an intrinsic disk emission lower than $\sim 20 \%$, i.e., the disk is mostly passive, radiating only through the reprocessing of the warm corona emission. As already suggested in the dedicated analysis of Mkn 509 (P13), a warm corona above a passive disk appears as a reasonable (from a statistical point of view) explanation of the soft X-ray excess in AGNs.

About half of our sample, however, are below the contour $\left.\frac{L_{d i s k, i n t r}}{L_{s}}\right|_{\min }=0 \%$ with an amplification ratio between 2 and 3 . As discussed in Sect. 2.1, this suggests a patchy warm corona. In the case of a corona above the accretion disk, we could use Eq. (19) to give some constraints on the corona "patchiness". Indeed, we have

$g=\frac{2}{A-\mathrm{e}^{-\tau}+2 \frac{L_{\text {disk,intr }}}{L_{s}}}$.

Assuming a passive disk (and large $\tau$ as estimated for the warm corona), we obtain $2 / 3<g<1$ for $A$ between 2 and 3 . In terms of a solid angle sustained by the warm corona, this translates to $4 \pi / 3<\Omega_{w c}<2 \pi$, i.e., a slightly patchy corona with respect to the slab.

By comparison, and as commonly observed, the hot corona parameters agree with large amplification ratios (mainly above 
P.-O. Petrucci et al.: Testing warm Comptonization models for the origin of the soft X-ray excess in AGNs
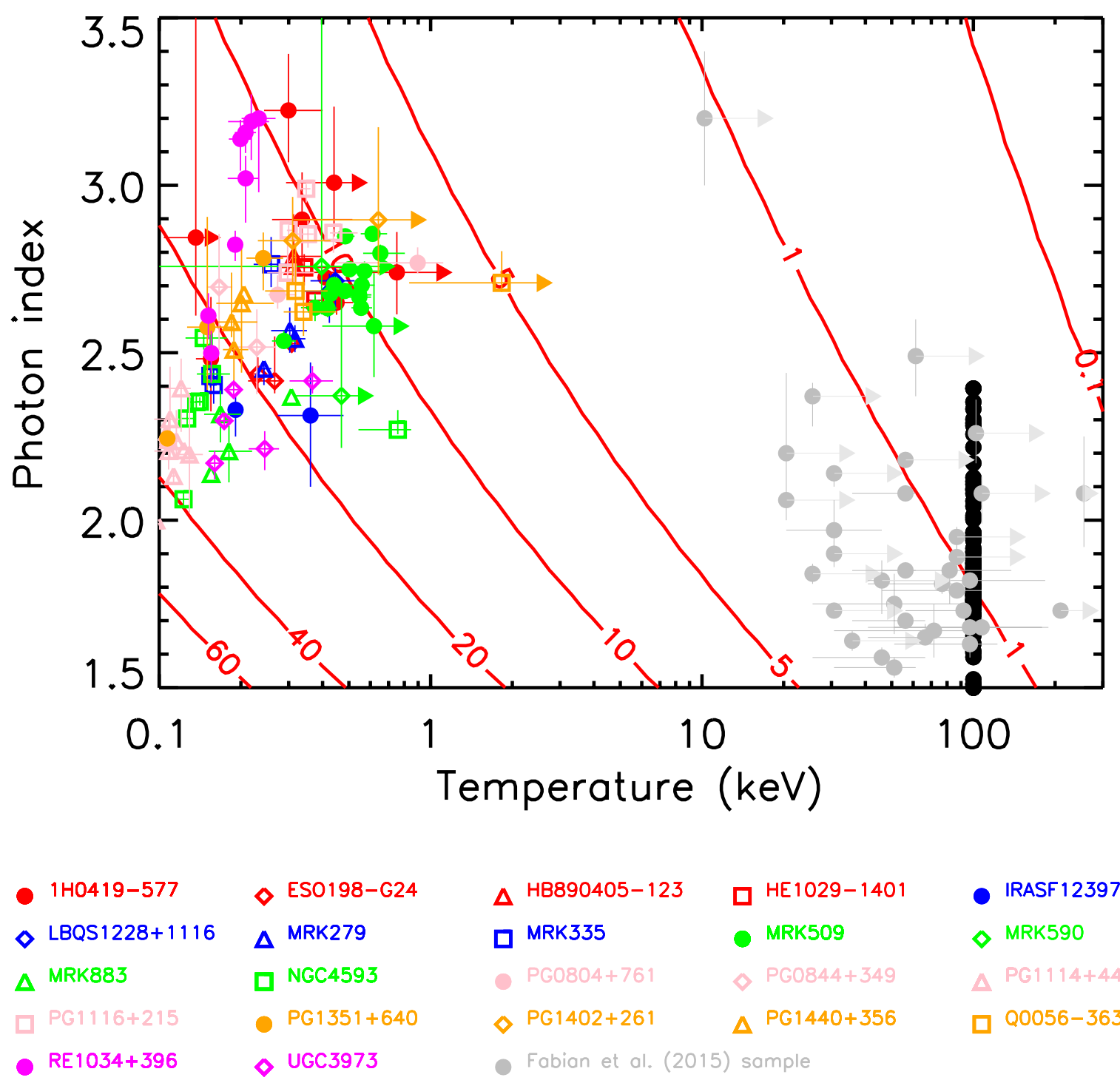

- IRASF $12397+3333$

$\checkmark$ MRK590

$\triangle \mathrm{PG} 1114+4.45$

Q0056-363

Fig. 5. Best fit temperature and photon index of the warm corona of the different objects of our sample. Different colored symbols are used for each object. They cluster in the left part of the figure, and agree with large $(>5)$ optical depths. For comparison, we have also reported the best fit photon indexes of the hot corona (black circles on the right). The temperature of the hot corona being fixed to $100 \mathrm{keV}$ in the fits, they are all aligned vertically. The gray filled circles correspond to the best fit parameters of the hot corona from Fabian et al. (2015). The hot corona parameters cluster in the bottom right part of the figure where the optical depth is close to unity.

10), the signature of a very photon-starved geometry. Using again the above expression (still assuming a passive disk but now with a corona optical depth $\tau=1$ ), we indeed find $g<0.2$ for $A>10$, or $\Omega_{h c}<2 \pi / 5$.

While crude and limited to the two-coronae framework, these estimates of the warm and hot coronae patchiness support a diskcoronae geometry where the hot corona is localized in the inner part of the accretion flow (where the hottest temperatures are expected to be reached), while the warm corona largely covers the outer part of an optically thick accretion disk (see, e.g., Fig. 10 in P13). The "lamp post" geometry, where the hot corona lies above the black hole and illuminates an accretion disk covered by a warm corona is also consistent with these estimates. This is different, however, from the geometries proposed by, e.g., Done et al. (2012) in which, by construction, the accretion disk is intrinsically luminous, which implies a smaller solid angle sustained by the warm corona.

\subsubsection{Correlation}

We do not find any significant correlations between the different best fit spectral parameters. We also check for correlations with the black hole mass and the UV/X-ray luminosity $L_{U V-X}$. We estimate the latter by integrating the flux of our unabsorbed SEDs. Using the estimate of the black hole mass for a large part of our sample (see Table B.1 and Bianchi et al. 2009 for the references; only HB890405-123 and LBQS1228+1116 have no BH mass estimates); we can then estimate $L_{U V-X} / L_{E d d}$. These values are listed in Table C.2. Interestingly, a strong correlation (with a linear Pearson correlation coefficient equal to 0.47 , which corresponds to a $>99 \%$ confidence level for its significance), is found between $\Gamma_{w c}$ and $L_{U V-X} / L_{E d d}$. It is shown in Fig. 9. A log-linear fit gives

$\Gamma_{w c}=(2.95 \pm 0.01)+(0.38 \pm 0.01) \log \left(\frac{L_{U V-X}}{L_{E d d}}\right)$ 

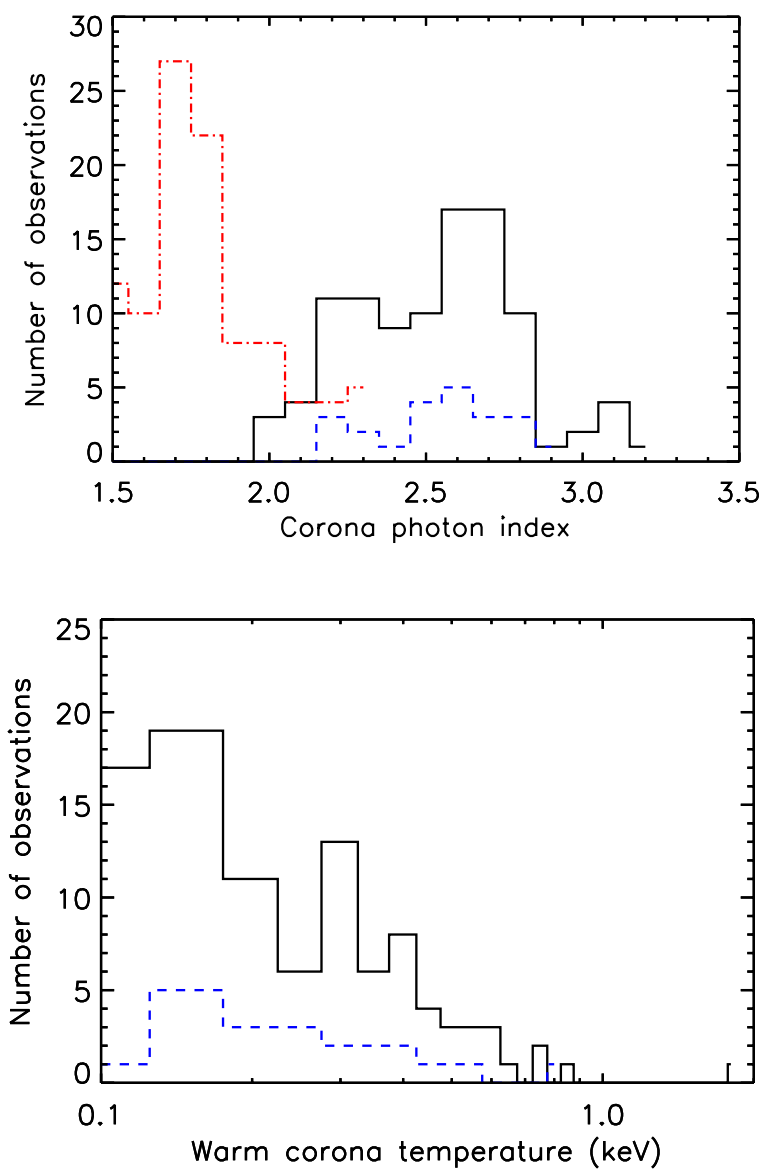

Fig. 6. Warm corona photon index $\Gamma_{w c}$ (top) and temperature $k T_{w c}$ (bottom) of the warm corona model for the different objects in our sample. The black solid histograms correspond to the whole observations. The dashed (blue) histogram corresponds to the average values of $\Gamma_{w c}$ and $k T_{w c}$ for each object. In the top figure, we have also overplotted the histogram of the hot corona photon index $\Gamma_{h c}$ (dot-dashed red line).

and is overplotted in the figure with a solid line. The slope value is low and indicates a rather smooth dependency. The standard deviation of all the points with respect to the best fit relation is $\sim 0.2$, slightly smaller than the dispersion of the warm corona photon index $(\sim 0.3)$. Since large $\Gamma_{w c}(>2.6)$ agree, in our two-coronae model, with larger intrinsic disk emission, this correlation suggests that high accretion rate systems would go with more intrinsic disk dissipation.

\section{Concluding remarks}

In this study, we discuss the theoretical expectations of the spectral parameters (i.e., photon index $\Gamma$ and temperature $k T_{e}$ ) of the Comptonization emission of a thermal corona in radiative equilibrium with the accretion disk. We detail how the corona optical depth $\tau$, its amplification factor $A$, and the minimal intrinsic disk emission $\left.\frac{L_{\text {disk,intr }}}{L_{s}}\right|_{\min }$ can be mapped in the $\Gamma-k T_{e}$ plane.

Then, we show that the spectral constraints published in the literature for the soft X-ray excess suggest an optically thick ( $\tau \sim$ 10-20), extended (amplification factor $A \simeq 2$ ) thermal corona in radiative equilibrium above a weakly dissipative disk. On the contrary, and as a well-known result, the hard X-ray emission agrees with an optically thin $(\tau \sim 1)$ and patchy $(A \gg 1)$ thermal corona.

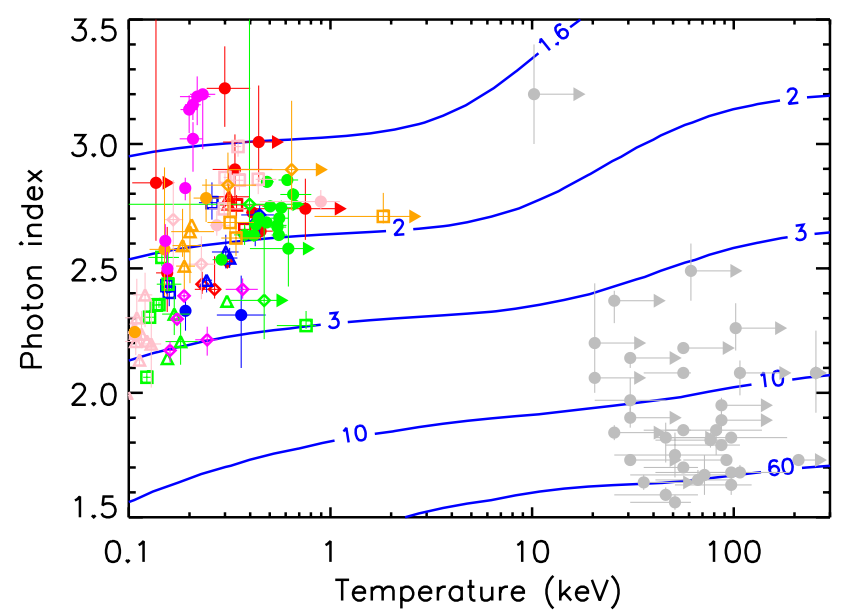

Fig. 7. Contours of the amplification factor $A$ in the corona spectral parameter $\Gamma-k T$ plane. The colored symbols and the gray filled circles are the same as in Fig. 1.

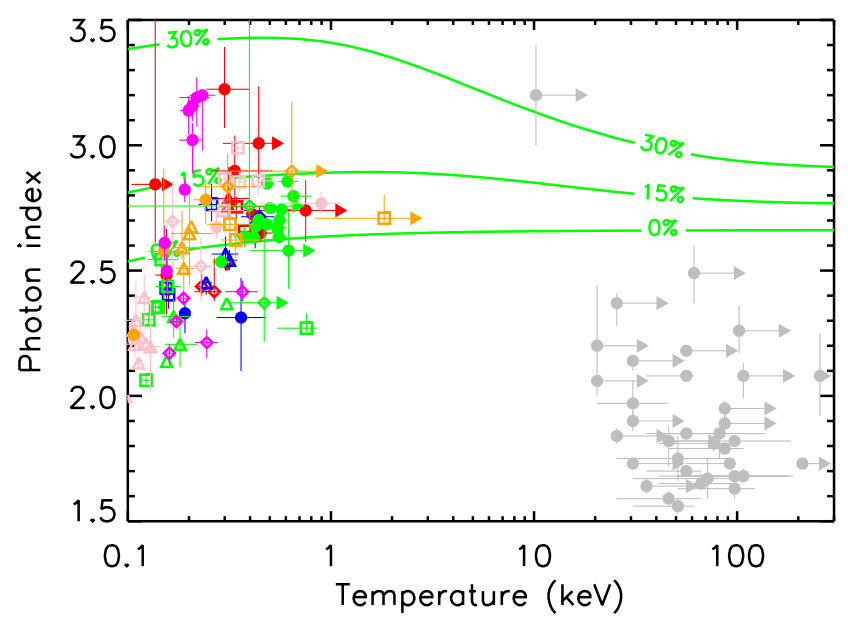

Fig. 8. Contours of the minimal fraction (in \%) of disk intrinsic emission $\left.\frac{L_{d i s k, i n t r}}{L_{s}}\right|_{\min }$ in the corona spectral parameter $\Gamma-k T$ plane. The colored symbols and the gray filled circles are the same as in Fig. 1.

To better test this two-coronae dichotomy, we apply the twocoronae model to a large sample of AGNs with simultaneous optical/UV and X-ray data from XMM-Newton. Our sample is composed of 22 AGNs and 100 ObsID. In the two-coronae model, the warm corona reproduces the entire optical/UV and soft X-ray emission, while the hot corona explains the highenergy ( $>2 \mathrm{keV}$ ) emission.

The two-coronae model gives a statistically reasonable fit to the optical/UV/X-ray data of our sample. Our best fit physical parameters for the warm corona indicate an optical depth $\tau \sim 10-40$ and an electron temperature $k T \sim 0.1-1 \mathrm{keV}$. In comparison, the hot corona is optically thin $(\tau \sim 1)$ and hot $(k T>20 \mathrm{keV})$. More interestingly, we confirm that the warm corona parameters agree well with a quite extended corona above a passive disk, i.e., most of the accretion power would be released in the warm corona, while the disk would only reradiate the emission coming from the corona. This result was first suggested by P13 who applied the two-coronae model to the monitoring of Mkn 509. The present study shows that this interpretation could apply to a large number of objects suggesting a geometry similar to Fig. 10 in P13 for the inner accretion flow. 


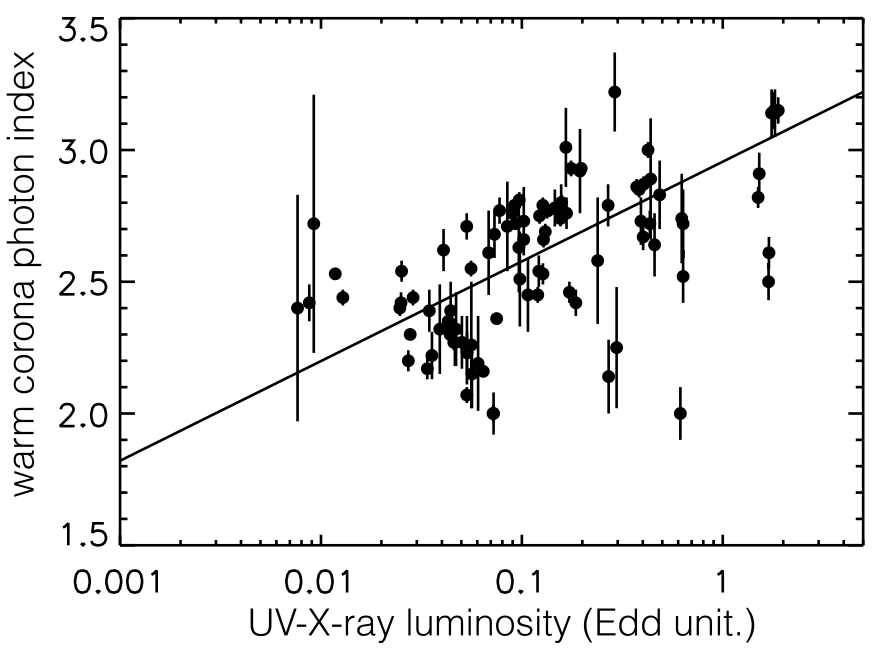

Fig. 9. Warm corona photon index versus UV/X-ray luminosity (in Eddington units) for all the observations of our sample, except those of HB890405-123 and LBQS1228+1116 for which we do not have black hole mass estimates. The solid line corresponds to the log-linear best fit.

If this interpretation seems qualitatively correct, the dispersion of the warm corona parameter values observed in the sample may indicate a true dispersion in the intrinsic disk and disk/corona geometry properties. Some objects, e.g., RE1034+396, show a quite steep warm corona photon index $\sim 3$ suggesting a more active underlying disk than the other sources, consistent with the known high $L / L_{E d d}$ in this object (see Table C.2, but also Jin et al. 2012a; Done et al. 2012). On the contrary, other objects, e.g., PG1114+445, have a quite hard warm corona photon index $\sim 2.2$ producing a weak "bend" (and consequently a weak soft X-ray excess) with respect to the hard $\mathrm{X}$-ray power law. Alternatives for the soft X-ray excess, e.g., blurred reflection and/or absorption, could play a significant role here (see similar discussion in Done et al. 2012; Jin et al. 2012a). A more careful modeling, which is beyond the scope of the present paper, would be required to test these hypotheses.

Our modeling of the hot and warm coronae with a onetemperature plasma is also certainly crude. A temperature distribution is expected, globally decreasing with radius. Thus, if the warm corona is radially elongated, as suggested by our results, it is probably not covering the entire disk and part of the observed optical emission could come from the outer part of the disk. Optical/UV reverberation studies also suggest that the accretion flow geometry could be quite complex and that the optical/UV emission could be produced by more than a single component (e.g., Gardner \& Done 2017 and references therein). The addition of a model component in the optical in our study would naturally imply a less extended warm corona, more localized in the inner (UV/Far UV emitting) region of the accretion flow, and, consequently, a larger intrinsic disk emission to explain the observed values of warm corona temperature and photon index. While we do not expect this effect to significantly change our conclusion, its precise estimate would also require a detailed modeling of the optical/UV emission, which is beyond the scope of the present paper given the spectral limitation due to the use of the XMM/OM large band filters.

Some radio-quiet AGNs show clear signatures of relativistic iron lines, while the absence of this component in the others could be due to a lack of statistics (e.g., Brenneman \& Reynolds 2009; de La Calle Pérez et al. 2010; Mantovani et al. 2016).
Relativistically blurred ionized reflection can produce significant emission in the soft X-rays and could even explain a large part of the soft X-ray excess (e.g., Crummy et al. 2006). Due to the additional complexity, our fits do not include a relativistic reflection. Our reduced $\chi^{2}$ are also reasonably good and do not require the addition of this component. If it were present, it would produce soft X-ray emission and, consequently, is expected to weaken the amount of soft X-ray emission coming from the warm corona. The fits would compensate with larger values of $\Gamma_{w c}$, moving our sample to the top of Fig. 1, i.e., the minimal fraction of the disk intrinsic emission would increase with respect to the present estimates. We do not expect, however, drastic changes in $\Gamma_{w c}$, and we believe that the data would still agree with a quite low fraction of disk intrinsic emission $(<50 \%)$, most of the accretion power being still released in the warm corona.

The presence of such a corona above the accretion disk may have important consequences, for example a direct impact on our understanding of the accretion disk vertical equilibrium and the expected spectral emission from such accretion flow or its capacity of producing outflows/jets. Moreover, it would certainly have some impacts on the spectral shape of the reflection component. In the case of a hot $(k T \simeq 100 \mathrm{keV})$ and optically thin $(\tau<1)$ corona, the reflection component is expected to be partly smeared out when crossing the corona, modifying the shape of the reflection hump and the iron line equivalent width measurements (e.g., Petrucci et al. 2001; Steiner et al. 2017). Similar effects are expected in the warm corona. Instead, the cooler temperature would produce the down scattering of the illuminating photons (e.g., the iron line should be redshifted), but some broadening should be present too. On the other hand, the warm corona temperature on the order of $0.5-1 \mathrm{keV}$ is such that several ionized lines should be produced and potentially observed. These are important signatures that have to be tested with accurate radiative transfer codes in order to confirm or rule out the presence of warm plasma in the inner part of the accretion flow.

Acknowledgements. We would like to thank the referee for the careful reading of the manuscript and useful comments that helped to improve its quality. This work is based on observations obtained with XMM-Newton, an ESA science mission with instruments and contributions directly funded by ESA Member States and the USA (NASA). We acknowledge financial support from the CNRS/INAF French/Italian PICS programme. P.O.P. acknowledges financial support from CNES and the French PNHE. P.O.P. and F.U. acknowledge support from the Italo/French Vinci programme. The research leading to these results has received funding from the European Union's Horizon 2020 Programme under AHEAD project (grant agreement no. 654215). S.B. and G.M. acknowledge financial support from the European Union Seventh Framework Programme (FP7/2007-2013) under grant agreement no. 31278. M.D. and M.C. acknowledge support from the ASI-INAF grant I/037/12/0.

\section{References}

Arnaud, K., Dorman, B., \& Gordon, C. 1999, XSPEC: An X-ray spectral fitting package, Astrophysics Source Code Library [record ascl:9910.005]

Arnaud, K. A. 1996, in Astronomical Data Analysis Software and Systems V, ASP Conf. Ser., 101, 17

Arnaud, K. A., Branduardi-Raymont, G., Culhane, J. L., et al. 1985, MNRAS, 217, 105

Beloborodov, A. M. 1999, in High Energy Processes in Accreting Black Holes, ASP Conf. Ser., 161, 295

Bianchi, S., Guainazzi, M., Matt, G., Fonseca Bonilla, N., \& Ponti, G. 2009, A\&A, 495, 421

Brenneman, L. W., \& Reynolds, C. S. 2009, ApJ, 702, 1367

Brenneman, L. W., Madejski, G., Fuerst, F., et al. 2014a, ApJ, 788, 61

Brenneman, L. W., Madejski, G., Fuerst, F., et al. 2014b, ApJ, 781, 83

Brocksopp, C., Starling, R. L. C., Schady, P., et al. 2006, MNRAS, 366, 953

Brunner, H., Mueller, C., Friedrich, P., et al. 1997, A\&A, 326, 885 
Cappi, M., De Marco, B., Ponti, G., et al. 2016, A\&A, 592, A27

Crummy, J., Fabian, A. C., Gallo, L., \& Ross, R. R. 2006, MNRAS, 365, 1067

de La Calle Pérez, I., Longinotti, A. L., Guainazzi, M., et al. 2010, A\&A, 524, A50

Done, C., Davis, S. W., Jin, C., Blaes, O., \& Ward, M. 2012, MNRAS, 420, 1848

Fabian, A. C., Lohfink, A., Kara, E., et al. 2015, MNRAS, 451, 4375

Gabriel, C., Denby, M., Fyfe, D. J., et al. 2004, in Astronomical Data Analysis Software and Systems (ADASS) XIII, eds. F. Ochsenbein, M. G. Allen, \& D. Egret, ASP Conf. Ser., 314, 759

Gallo, L. C., Tanaka, Y., Boller, T., et al. 2004, MNRAS, 353, 1064

García, J., \& Kallman, T. R. 2010, ApJ, 718, 695

Gardner, E., \& Done, C. 2017, MNRAS, 470, 3591

Gelbord, J., Gronwall, C., Grupe, D., Vanden Berk, D., \& Wu, J. 2015, ArXiv e-prints [arXiv: 1505.05248]

Gierlinski, M., \& Done, C. 2004, MNRAS, 349, L7

Güver, T., \& Özel, F. 2009, MNRAS, 400, 2050

Haardt, F. 1993, ApJ, 413, 680

Haardt, F., \& Maraschi, L. 1991, ApJ, 380, L51

Haardt, F., \& Maraschi, L. 1993, ApJ, 413, 507

Haardt, F., Maraschi, L., \& Ghisellini, G. 1994, ApJ, 432, L95

Henri, G., \& Petrucci, P. O. 1997, A\&A, 326, 87

Jin, C., Ward, M., \& Done, C. 2012a, MNRAS, 425, 907

Jin, C., Ward, M., Done, C., \& Gelbord, J. 2012b, MNRAS, 420, 1825

Kaastra, J. S., Petrucci, P. O., Cappi, M., et al. 2011, A\&A, 534, A36

Kaastra, J. S., Kriss, G. A., Cappi, M., et al. 2014, Science, 345, 64

Kalberla, P. M. W., Burton, W. B., Hartmann, D., et al. 2005, A\&A, 440, 775

Kubota, A., \& Done, C. 2016, MNRAS, 458, 4238

Laor, A., Fiore, F., Elvis, M., Wilkes, B. J., \& McDowell, J. C. 1997, ApJ, 477, 93
Magdziarz, P., Blaes, O. M., Zdziarski, A. A., Johnson, W. N., \& Smith, D. A. 1998, MNRAS, 301, 179

Mantovani, G., Nandra, K., \& Ponti, G. 2016, MNRAS, 458, 4198

Mason, K. O., Breeveld, A., Much, R., et al. 2001, A\&A, 365, L36

Matt, G., Marinucci, A., Guainazzi, M., et al. 2014, MNRAS, 439, 3016

Mehdipour, M., Branduardi-Raymont, G., Kaastra, J. S., et al. 2011, A\&A, 534, A39

Mehdipour, M., Kaastra, J. S., Kriss, G. A., et al. 2015, A\&A, 575, A22

Page, K. L., Schartel, N., Turner, M. J. L., \& O'Brien, P. T. 2004, MNRAS, 352, 523

Page, M. J., Brindle, C., Talavera, A., et al. 2012, MNRAS, 426, 903

Petrucci, P. O., Merloni, A., Fabian, A., Haardt, F., \& Gallo, E. 2001, MNRAS, 328, 501

Petrucci, P.-O., Paltani, S., Malzac, J., et al. 2013, A\&A, 549, A73

Piconcelli, E., Jimenez-Bailón, E., Guainazzi, M., et al. 2004, MNRAS, 351, 161

Ponti, G., Papadakis, I., Bianchi, S., et al. 2012, A\&A, 542, A83

Read, A. M., \& Ponman, T. J. 2003, A\&A, 409, 395

Różańska, A., Malzac, J., Belmont, R., Czerny, B., \& Petrucci, P.-O. 2015, A\&A, 580, A77

Sako, M., Kahn, S. M., Branduardi-Raymont, G., et al. 2003, ApJ, 596, 114

Schartel, N., Green, P. J., Anderson, S. F., et al. 1996, MNRAS, 283, 1015

Shakura, N. I., \& Sunyaev, R. A. 1973, A\&A, 24, 337

Steiner, J. F., García, J. A., Eikmann, W., et al. 2017, ApJ, 836, 119

Stern, B. E., Poutanen, J., Svensson, R., Sikora, M., \& Begelman, M. C. 1995, ApJ, 449, L13

Strüder, L., Briel, U., Dennerl, K., et al. 2001, A\&A, 365, L18

Véron-Cetty, M.-P., \& Véron, P. 2010, A\&A, 518, A10

Walter, R., \& Fink, H. H. 1993, A\&A, 274, 105

Zdziarski, A. A., Johnson, W. N., \& Magdziarz, P. 1996, MNRAS, 283, 193

Życki, P. T., Done, C., \& Smith, D. A. 1999, MNRAS, 309, 561 


\section{Appendix A: Spectral index of the Compton emission from a slab and optically thick corona above a passive disk}

We demonstrate here that the constraints on the photon rate and the amplification factor (see Sect. 2.1) impose a corona photon index close to 2.6. For simplification, let us assume the corona to be spherical with radius $R$, and the disk emission spectral density to have a blackbody distribution (instead of a disk blackbody distribution) characterized by a temperature $T_{b b}$, homogeneously distributed inside the corona. This is different from a slab geometry, where the disk emission comes from below the corona. For a spherical, optically thick corona, Eqs. (4) and (15) become

$$
\dot{n}_{s} \simeq \dot{n}_{o b s}
$$$$
L_{s} \simeq L_{o b s} \text {. }
$$

Then, from the integration of the blackbody distribution, the disk photon rate $\dot{n}_{s}$ and luminosity $L_{s}$ are given by

$$
\begin{aligned}
\dot{n}_{s} & =\frac{16 \pi \zeta(3) k^{3}}{h^{3} c^{3}} T_{b b}^{3} 4 \pi R^{2} c \\
& =2.029 \times 10^{7} T_{b b}^{3} 4 \pi R^{2} c \quad \mathrm{ph} \cdot \mathrm{s}^{-1}
\end{aligned}
$$

with $\zeta(3)$ the Apery constant (equal to $\sim 1.2$ ).

On the other hand, approximating the Compton corona spectrum by a simple power law, $F_{E}=N_{0} E^{-\Gamma}$, between $E_{b b}=k T_{b b}$ and a cutoff energy $E_{c}$, the observed corona photon rate $\dot{n}_{o b s}$ and luminosity $L_{o b s}$ are given by

$\dot{n}_{o b s}=\frac{N_{0}}{1-\Gamma}\left(E_{c}^{1-\Gamma}-E_{b b}^{1-\Gamma}\right)$
$L_{o b s}=\frac{N_{0}}{2-\Gamma}\left(E_{c}^{2-\Gamma}-E_{b b}^{2-\Gamma}\right)$.

Combining these different equations (from A.1 to A.8) and assuming $E_{c} \gg E_{b b}$ (i.e., neglecting $E_{c}^{1-\Gamma}$ and $E_{c}^{2-\Gamma}$ with respect to $E_{b b}^{1-\Gamma}$ and $E_{b b}^{1-\Gamma}$, respectively) we obtain a simple relation on $\Gamma$ :

(A.4) $\frac{1-\Gamma}{2-\Gamma} \simeq 2.7$ i.e., $\Gamma \simeq 2.6$. 


\section{Appendix B: The sample}

Table B.1. List of objects in our sample.

\begin{tabular}{|c|c|c|c|c|c|c|c|}
\hline Source & RA & Dec & Redshift & $\log M_{b h}$ & $E(B-V)$ & ObsID & Available OM filters \\
\hline 1H0419-577 & 66.5030 & -57.2002 & 0.1040 & 8.580 & 0.0296 & $\begin{array}{l}0148000201 \\
0148000301 \\
0148000401 \\
0148000501 \\
0148000601 \\
0604720301 \\
0604720401 \\
0112600401\end{array}$ & $\begin{array}{c}\mathrm{U}, \mathrm{B}, \mathrm{V}, \mathrm{W} 1, \mathrm{~W} 2 \\
\mathrm{U}, \mathrm{B}, \mathrm{V}, \mathrm{W} 1, \mathrm{~W} 2 \\
\mathrm{U}, \mathrm{B}, \mathrm{V}, \mathrm{W} 1, \mathrm{~W} 2 \\
\mathrm{U}, \mathrm{B}, \mathrm{V}, \mathrm{W} 1, \mathrm{~W} 2 \\
\mathrm{U}, \mathrm{B}, \mathrm{V}, \mathrm{W} 1, \mathrm{~W} 2 \\
\mathrm{~B}, \mathrm{~W} 1, \mathrm{M} 2, \mathrm{~W} 2 \\
\mathrm{~B}, \mathrm{~W} 1, \mathrm{M} 2, \mathrm{~W} 2 \\
\mathrm{~W} 1\end{array}$ \\
\hline ESO198-G24 & 39.5818 & -52.1923 & 0.0455 & 8.48 & 0.0456 & $\begin{array}{l}0305370101 \\
0112910101 \\
0067190101\end{array}$ & $\begin{array}{c}\mathrm{U}, \mathrm{B}, \mathrm{W} 1, \mathrm{M} 2, \mathrm{~W} 2 \\
\mathrm{U} \\
\mathrm{W} 1, \mathrm{~W} 2\end{array}$ \\
\hline HB890405-123 & 61.9517 & -12.1935 & 0.5725 & - & 0.0556 & $\begin{array}{l}0202210401 \\
0202210301\end{array}$ & $\begin{array}{l}\mathrm{U}, \mathrm{W} 1, \mathrm{~W} 2 \\
\mathrm{U}, \mathrm{W} 1, \mathrm{~W} 2\end{array}$ \\
\hline HE1029-1401 & 157.976 & -14.2808 & 0.0858 & 8.73 & 0.0954 & $\begin{array}{l}0203770101 \\
0110950101\end{array}$ & $\begin{array}{c}\mathrm{U}, \mathrm{V}, \mathrm{W} 1, \mathrm{M} 2, \mathrm{~W} 2 \\
\mathrm{~W} 2\end{array}$ \\
\hline IRASF12397+3333 & 190.544 & 33.2840 & 0.0435 & 6.66 & 0.0194 & $\begin{array}{l}0202180301 \\
0202180201\end{array}$ & $\begin{array}{c}\mathrm{U}, \mathrm{B}, \mathrm{W} 1, \mathrm{M} 2 \\
\mathrm{U}, \mathrm{B}, \mathrm{V}, \mathrm{W} 1, \mathrm{M} 2, \mathrm{~W} 2\end{array}$ \\
\hline LBQS1228+1116 & 187.725 & 11.0031 & 0.2362 & - & 0.0313 & $\begin{array}{l}0306630201 \\
0306630101\end{array}$ & $\begin{array}{c}\mathrm{U}, \mathrm{B}, \mathrm{V}, \mathrm{W} 1, \mathrm{M} 2, \mathrm{~W} 2 \\
\mathrm{U}, \mathrm{B}, \mathrm{V}, \mathrm{M} 2, \mathrm{~W} 2\end{array}$ \\
\hline MRK279 & 208.264 & 69.3082 & 0.0304 & 7.54 & 0.0259 & $\begin{array}{l}0302480501 \\
0302480601 \\
0302480401\end{array}$ & $\begin{array}{l}\mathrm{U}, \mathrm{W} 1, \mathrm{M} 2, \mathrm{~W} 2 \\
\mathrm{U}, \mathrm{W} 1, \mathrm{M} 2, \mathrm{~W} 2 \\
\mathrm{U}, \mathrm{W} 1, \mathrm{M} 2, \mathrm{~W} 2\end{array}$ \\
\hline MRK335 & 1.58130 & 20.2029 & 0.0257 & 7.15 & 0.0582 & $\begin{array}{l}0600540601 \\
0600540501 \\
0510010701\end{array}$ & $\begin{array}{c}\mathrm{U}, \mathrm{B}, \mathrm{V}, \mathrm{W} 1, \mathrm{M} 2, \mathrm{~W} 2 \\
\mathrm{U}, \mathrm{B}, \mathrm{V}, \mathrm{W} 1, \mathrm{M} 2, \mathrm{~W} 2 \\
\mathrm{U}, \mathrm{B}, \mathrm{W} 1, \mathrm{M} 2, \mathrm{~W} 2\end{array}$ \\
\hline MRK509 & 311.0405 & -10.7234 & 0.0343 & 8.16 & 0.0600 & $\begin{array}{l}0601390201 \\
0601390301 \\
0601390401 \\
0601390501 \\
0601390601 \\
0601390701 \\
0601390801 \\
0601390901 \\
0601391001 \\
0601391101 \\
0306090101 \\
0306090201 \\
0306090301 \\
0306090401 \\
0130720101 \\
0130720201\end{array}$ & $\begin{array}{c}\mathrm{U}, \mathrm{B}, \mathrm{V}, \mathrm{W} 1, \mathrm{M} 2, \mathrm{~W} 2 \\
\mathrm{U}, \mathrm{B}, \mathrm{V}, \mathrm{W} 1, \mathrm{M} 2, \mathrm{~W} 2 \\
\mathrm{U}, \mathrm{B}, \mathrm{V}, \mathrm{W} 1, \mathrm{M} 2, \mathrm{~W} 2 \\
\mathrm{U}, \mathrm{B}, \mathrm{V}, \mathrm{W} 1, \mathrm{M} 2, \mathrm{~W} 2 \\
\mathrm{U}, \mathrm{B}, \mathrm{V}, \mathrm{W} 1, \mathrm{M} 2, \mathrm{~W} 2 \\
\mathrm{U}, \mathrm{B}, \mathrm{V}, \mathrm{W} 1, \mathrm{M} 2, \mathrm{~W} 2 \\
\mathrm{U}, \mathrm{B}, \mathrm{V}, \mathrm{W} 1, \mathrm{M} 2, \mathrm{~W} 2 \\
\mathrm{U}, \mathrm{B}, \mathrm{V}, \mathrm{W} 1, \mathrm{M} 2, \mathrm{~W} 2 \\
\mathrm{U}, \mathrm{B}, \mathrm{V}, \mathrm{W} 1, \mathrm{M} 2, \mathrm{~W} 2 \\
\mathrm{U}, \mathrm{B}, \mathrm{V}, \mathrm{W} 1, \mathrm{M} 2, \mathrm{~W} 2 \\
\mathrm{~W} 1 \\
\mathrm{M} 2, \mathrm{~W} 2 \\
\mathrm{~W} 1, \mathrm{M} 2, \mathrm{~W} 2 \\
\mathrm{~W} 1, \mathrm{M} 2, \mathrm{~W} 2 \\
\mathrm{M} 2, \mathrm{~W} 2 \\
\mathrm{U}, \mathrm{W} 1, \mathrm{~W} 2\end{array}$ \\
\hline MRK590 & 33.6398 & -0.766600 & 0.0263 & 7.68 & 0.0391 & $\begin{array}{l}0109130301 \\
0201020201\end{array}$ & $\begin{array}{c}\mathrm{U}, \mathrm{B}, \mathrm{V}, \mathrm{W} 1, \mathrm{M} 2, \mathrm{~W} 2 \\
\mathrm{U}, \mathrm{B}, \mathrm{W} 1, \mathrm{M} 2, \mathrm{~W} 2\end{array}$ \\
\hline MRK883 & 247.470 & 24.4439 & 0.0374 & 7.28 & 0.0579 & $\begin{array}{l}0652550201 \\
0302260701 \\
0302261001 \\
0302260101\end{array}$ & $\begin{array}{c}\mathrm{U}, \mathrm{B}, \mathrm{W} 1 \\
\mathrm{U}, \mathrm{B}, \mathrm{W} 1, \mathrm{M} 2, \mathrm{~W} 2 \\
\mathrm{U}, \mathrm{B}, \mathrm{W} 1, \mathrm{M} 2, \mathrm{~W} 2 \\
\mathrm{U}, \mathrm{B}, \mathrm{W} 1, \mathrm{M} 2, \mathrm{~W} 2\end{array}$ \\
\hline NGC4593 & 189.9142 & -5.3442 & 0.0090 & 6.729 & 0.0337 & $\begin{array}{l}0740920201 \\
0740920301 \\
0740920401 \\
0740920501 \\
0740920601 \\
0109970101 \\
0059830101\end{array}$ & $\begin{array}{c}\mathrm{U}, \mathrm{W} 1, \mathrm{~W} 2 \\
\mathrm{U}, \mathrm{W} 1, \mathrm{~W} 2 \\
\mathrm{U}, \mathrm{W} 1, \mathrm{~W} 2 \\
\mathrm{U}, \mathrm{W} 1, \mathrm{~W} 2 \\
\mathrm{U}, \mathrm{W} 1, \mathrm{~W} 2 \\
\mathrm{~W} 1, \mathrm{~W} 2 \\
\mathrm{~W} 1, \mathrm{M} 2, \mathrm{~W} 2\end{array}$ \\
\hline PG0804+761 & 122.744 & 76.0451 & 0.1000 & 8.24 & 0.0435 & 0605110101 & $\mathrm{U}, \mathrm{V}, \mathrm{W} 1, \mathrm{M} 2$ \\
\hline
\end{tabular}

Notes. The reddening $E(B-V)$ is calculated from the Galactic extinction following Güver \& Özel (2009) unless otherwise specified. 
Table B.1. continued.

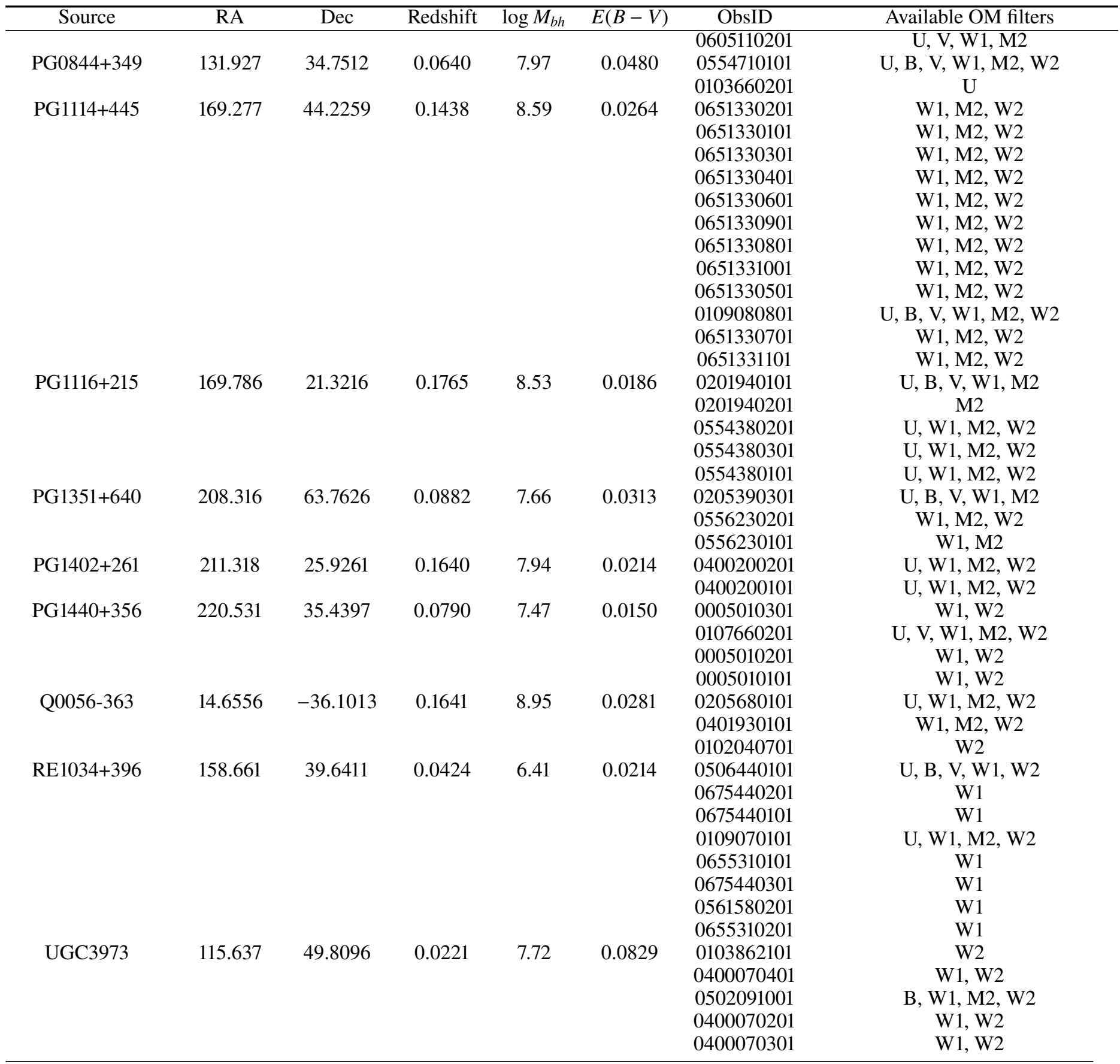


Appendix C: Best fit parameter and bolomeric luminosity values

Table C.1. Best fit parameters of each observation of our sample.

\begin{tabular}{|c|c|c|c|c|c|c|c|}
\hline Source & ObsID & $\Gamma_{w c}$ & $\begin{array}{c}k T_{w c} \\
(\mathrm{keV})\end{array}$ & $\Gamma_{h c}$ & $\begin{array}{l}k T_{b b} \\
(\mathrm{eV})\end{array}$ & $\chi_{\text {red }}^{2}$ & dof \\
\hline \multirow[t]{8}{*}{ 1H0419-577 } & 0148000201 & $2.48 \pm 0.17$ & $0.16 \pm 0.01$ & $1.50 \pm 0.04$ & $3.5 \pm 1.4$ & 1.29 & 196 \\
\hline & 0148000301 & $2.84 \pm 0.44$ & $0.14 \pm 4.95$ & $1.76 \pm 0.08$ & $4.5 \pm 2.9$ & 1.32 & 175 \\
\hline & 0148000401 & $3.22 \pm 0.16$ & $0.30 \pm 0.08$ & $1.77 \pm 0.06$ & $13.3 \pm 2.0$ & 0.98 & 201 \\
\hline & 0148000501 & $3.01 \pm 0.19$ & $0.44 \pm 4.85$ & $1.68 \pm 0.13$ & $7.3 \pm 2.3$ & 1.13 & 197 \\
\hline & 0148000601 & $2.74 \pm 0.12$ & $0.75 \pm 4.85$ & $1.63 \pm 0.13$ & $3.9 \pm 0.8$ & 0.96 & 197 \\
\hline & 0604720301 & $2.72 \pm 0.03$ & $0.41 \pm 0.03$ & $1.61 \pm 0.03$ & $5.8 \pm 0.3$ & 1.29 & 221 \\
\hline & 0604720401 & $2.65 \pm 0.04$ & $0.45 \pm 0.06$ & $1.59 \pm 0.04$ & $4.4 \pm 0.3$ & 1.10 & 221 \\
\hline & 0112600401 & $2.90 \pm 0.14$ & $0.34 \pm 0.13$ & $1.85 \pm 0.08$ & $6.3 \pm 1.2$ & 0.84 & 181 \\
\hline \multirow[t]{3}{*}{ ESO198-G24 } & 0305370101 & $2.53 \pm 0.02$ & $0.31 \pm 0.02$ & $1.72 \pm 0.02$ & $3.0^{f}$ & 1.55 & 226 \\
\hline & 0112910101 & $2.42 \pm 0.09$ & $0.27 \pm 0.03$ & $1.85 \pm 0.04$ & - & 1.16 & 184 \\
\hline & 0067190101 & $2.44 \pm 0.04$ & $0.23 \pm 0.01$ & $1.83 \pm 0.01$ & - & 1.30 & 222 \\
\hline \multirow[t]{2}{*}{ HB890405-123 } & 0202210401 & $2.79 \pm 0.08$ & $0.31 \pm 0.06$ & $1.76 \pm 0.02$ & $2.1 \pm 0.2$ & 1.10 & 219 \\
\hline & 0202210301 & $2.76 \pm 0.03$ & $0.31 \pm 0.02$ & $1.75 \pm 0.03$ & $2.1 \pm 0.1$ & 0.96 & 218 \\
\hline \multirow[t]{2}{*}{ HE1029-1401 } & 0203770101 & $2.66 \pm 0.01$ & $0.38 \pm 0.03$ & $1.81 \pm 0.03$ & $2.5^{f}$ & 1.33 & 226 \\
\hline & 0110950101 & $2.75 \pm 0.05$ & $0.34 \pm 0.11$ & $1.95 \pm 0.09$ & - & 1.06 & 168 \\
\hline \multirow[t]{2}{*}{ IRASF12397+3333 } & 0202180301 & $2.31 \pm 0.19$ & $0.36 \pm 0.10$ & $2.06 \pm 0.14$ & $8.0 \pm 3.4$ & 0.99 & 159 \\
\hline & 0202180201 & $2.33 \pm 0.06$ & $0.19 \pm 0.01$ & $2.30 \pm 0.03$ & $20.0 \pm 0.5$ & 1.32 & 226 \\
\hline \multirow[t]{2}{*}{ LBQS1228+1116 } & 0306630201 & $2.71 \pm 0.06$ & $0.45 \pm 0.08$ & $1.86 \pm 0.08$ & $2.5 \pm 0.3$ & 1.20 & 191 \\
\hline & 0306630101 & $2.68 \pm 0.07$ & $0.42 \pm 0.08$ & $1.84 \pm 0.10$ & $2.3 \pm 0.4$ & 1.06 & 177 \\
\hline \multirow[t]{3}{*}{ MRK279 } & 0302480501 & $2.45 \pm 0.03$ & $0.24 \pm 0.01$ & $1.79 \pm 0.02$ & $2.8 \pm 0.3$ & 1.31 & 224 \\
\hline & 0302480601 & $2.57 \pm 0.07$ & $0.30 \pm 0.05$ & $1.86 \pm 0.03$ & $3.4 \pm 0.6$ & 0.98 & 224 \\
\hline & 0302480401 & $2.54 \pm 0.04$ & $0.32 \pm 0.03$ & $1.85 \pm 0.02$ & $3.3 \pm 0.4$ & 1.37 & 224 \\
\hline \multirow[t]{3}{*}{ MRK335 } & 0600540601 & $2.43 \pm 0.04$ & $0.15 \pm 0.00$ & $1.94 \pm 0.03$ & $2.6 \pm 0.3$ & 1.50 & 224 \\
\hline & 0600540501 & $2.40 \pm 0.05$ & $0.16 \pm 0.01$ & $1.96 \pm 0.03$ & $2.8 \pm 0.4$ & 1.41 & 220 \\
\hline & 0510010701 & $2.76 \pm 0.07$ & $0.26 \pm 0.04$ & $1.52 \pm 0.06$ & $2.2 \pm 0.3$ & 1.74 & 172 \\
\hline \multirow[t]{16}{*}{ MRK509 } & 0601390201 & $2.74 \pm 0.03$ & $0.57 \pm 0.07$ & $1.75 \pm 0.03$ & $4.0 \pm 0.2$ & 1.06 & 225 \\
\hline & 0601390301 & $2.67 \pm 0.02$ & $0.55 \pm 0.05$ & $1.76 \pm 0.03$ & $3.5 \pm 0.2$ & 1.33 & 225 \\
\hline & 0601390401 & $2.80 \pm 0.03$ & $0.65 \pm 0.12$ & $1.77 \pm 0.04$ & $5.0 \pm 0.2$ & 1.24 & 225 \\
\hline & 0601390501 & $2.86 \pm 0.02$ & $0.61 \pm 0.07$ & $1.72 \pm 0.03$ & $5.6 \pm 0.2$ & 1.49 & 225 \\
\hline & 0601390601 & $2.85 \pm 0.02$ & $0.48 \pm 0.04$ & $1.80 \pm 0.03$ & $6.2 \pm 0.2$ & 1.46 & 225 \\
\hline & 0601390701 & $2.75 \pm 0.02$ & $0.50 \pm 0.05$ & $1.78 \pm 0.03$ & $4.6 \pm 0.2$ & 1.76 & 225 \\
\hline & 0601390801 & $2.70 \pm 0.02$ & $0.56 \pm 0.06$ & $1.77 \pm 0.03$ & $4.2 \pm 0.2$ & 1.32 & 225 \\
\hline & 0601390901 & $2.63 \pm 0.02$ & $0.55 \pm 0.05$ & $1.78 \pm 0.03$ & $3.6 \pm 0.2$ & 1.24 & 225 \\
\hline & 0601391001 & $2.70 \pm 0.02$ & $0.44 \pm 0.03$ & $1.80 \pm 0.02$ & $4.1 \pm 0.2$ & 1.51 & 225 \\
\hline & 0601391101 & $2.68 \pm 0.02$ & $0.48 \pm 0.04$ & $1.76 \pm 0.02$ & $3.9 \pm 0.2$ & 1.35 & 225 \\
\hline & 0306090101 & $2.58 \pm 0.16$ & $0.62 \pm 4.80$ & $1.71 \pm 0.16$ & $2.9 \pm 0.9$ & 1.07 & 191 \\
\hline & 0306090201 & $2.67 \pm 0.02$ & $0.55 \pm 0.05$ & $1.72 \pm 0.03$ & $5.0 \pm 0.2$ & 1.20 & 222 \\
\hline & 0306090301 & $2.68 \pm 0.03$ & $0.43 \pm 0.04$ & $1.78 \pm 0.03$ & $5.1 \pm 0.3$ & 1.24 & 223 \\
\hline & 0306090401 & $2.63 \pm 0.03$ & $0.42 \pm 0.03$ & $1.74 \pm 0.02$ & $3.7 \pm 0.2$ & 1.35 & 223 \\
\hline & 0130720101 & $2.60 \pm 0.01$ & $0.32 \pm 0.05$ & $1.70 \pm 0.03$ & $<2.5$ & 1.20 & 222 \\
\hline & 0130720201 & $2.63 \pm 0.04$ & $0.38 \pm 0.05$ & $1.78 \pm 0.03$ & $3.4 \pm 0.3$ & 1.67 & 223 \\
\hline \multirow[t]{2}{*}{ MRK590 } & 0109130301 & $2.37 \pm 0.23$ & $0.47 \pm 4.84$ & $1.68 \pm 0.18$ & $1.1 \pm 0.8$ & 0.83 & 144 \\
\hline & 0201020201 & $2.76 \pm 0.44$ & $0.40 \pm 4.98$ & $1.77 \pm 0.03$ & $1.0 \pm 0.1$ & 1.17 & 225 \\
\hline \multirow[t]{4}{*}{ MRK883 } & 0652550201 & $2.32 \pm 0.08$ & $0.17 \pm 0.03$ & $1.87 \pm 0.11$ & $1.0 \pm 0.1$ & 1.17 & 128 \\
\hline & 0302260701 & $2.21 \pm 0.08$ & $0.18 \pm 0.03$ & $1.74 \pm 0.14$ & $1.0 \pm 0.2$ & 1.26 & 104 \\
\hline & 0302261001 & $2.14 \pm 0.21$ & $0.16 \pm 0.11$ & $1.91 \pm 0.23$ & $1.0 \pm 0.2$ & 1.16 & 114 \\
\hline & 0302260101 & $2.37 \pm 0.10$ & $0.31 \pm 0.16$ & $1.50 \pm 0.06$ & $1.0 \pm 0.2$ & 1.26 & 95 \\
\hline \multirow[t]{7}{*}{ NGC4593 } & 0740920201 & $2.27 \pm 0.04$ & $0.76 \pm 0.15$ & $1.50 \pm 0.10$ & $1.7^{f}$ & 1.61 & 151 \\
\hline & 0740920301 & $2.54 \pm 0.04$ & $0.15 \pm 0.03$ & $1.69 \pm 0.03$ & - & 1.13 & 143 \\
\hline & 0740920401 & $2.44 \pm 0.03$ & $0.16 \pm 0.02$ & $1.78 \pm 0.03$ & - & 1.45 & 144 \\
\hline & 0740920501 & $2.30 \pm 0.02$ & $0.13 \pm 0.01$ & $1.84 \pm 0.02$ & - & 1.34 & 149 \\
\hline & 0740920601 & $2.35 \pm 0.02$ & $0.14 \pm 0.01$ & $1.88 \pm 0.02$ & - & 1.34 & 154 \\
\hline & 0109970101 & $2.06 \pm 0.03$ & $0.12 \pm 0.01$ & $1.87 \pm 0.02$ & - & 1.27 & 222 \\
\hline & 0059830101 & $2.35 \pm 0.01$ & $0.14 \pm 0.00$ & $1.89 \pm 0.01$ & - & 2.17 & 224 \\
\hline
\end{tabular}

Notes. The (f) means that the parameter is frozen during the fitting procedure. 
Table C.1. continued.

\begin{tabular}{|c|c|c|c|c|c|c|c|}
\hline Source & ObsID & $\Gamma_{w c}$ & $\begin{array}{c}k T_{w c} \\
(\mathrm{keV})\end{array}$ & $\Gamma_{h c}$ & $\begin{array}{l}k T_{b b} \\
(\mathrm{eV})\end{array}$ & $\chi_{\text {red }}^{2}$ & dof \\
\hline \multirow[t]{2}{*}{ PG0804+761 } & 0605110101 & $2.67 \pm 0.05$ & $0.27 \pm 0.03$ & $2.00 \pm 0.07$ & $2.5 \pm 0.3$ & 1.18 & 216 \\
\hline & 0605110201 & $2.77 \pm 0.04$ & $0.90 \pm 0.33$ & $1.50 \pm 0.20$ & $3.0 \pm 0.2$ & 1.33 & 211 \\
\hline \multirow[t]{2}{*}{ PG0844+349 } & 0554710101 & $2.70 \pm 0.17$ & $0.17 \pm 0.02$ & $1.50 \pm 0.02$ & $2.6 \pm 1.0$ & 1.05 & 85 \\
\hline & 0103660201 & $2.52 \pm 0.13$ & $0.23 \pm 0.04$ & $2.13 \pm 0.07$ & $2.4 \pm 0.9$ & 1.14 & 182 \\
\hline \multirow[t]{12}{*}{ PG1114+445 } & 0651330201 & $2.00 \pm 0.06$ & $0.10 \pm 0.01$ & $1.76 \pm 0.14$ & $2.6^{f}$ & 0.82 & 115 \\
\hline & 0651330101 & $2.24 \pm 0.13$ & $0.10 \pm 0.01$ & $1.51 \pm 0.05$ & - & 1.09 & 158 \\
\hline & 0651330301 & $2.21 \pm 0.25$ & $0.12 \pm 0.02$ & $1.68 \pm 0.13$ & - & 1.16 & 140 \\
\hline & 0651330401 & $2.00 \pm 0.03$ & $0.10 \pm 0.01$ & $1.96 \pm 0.09$ & - & 1.10 & 168 \\
\hline & 0651330601 & $2.13 \pm 0.02$ & $0.11 \pm 0.00$ & $1.76 \pm 0.06$ & - & 1.11 & 177 \\
\hline & 0651330901 & $2.25 \pm 0.10$ & $0.11 \pm 0.01$ & $1.74 \pm 0.09$ & - & 1.09 & 171 \\
\hline & 0651330801 & $2.30 \pm 0.14$ & $0.11 \pm 0.01$ & $1.65 \pm 0.07$ & - & 1.22 & 170 \\
\hline & 0651331001 & $2.39 \pm 0.11$ & $0.12 \pm 0.01$ & $1.67 \pm 0.08$ & - & 0.82 & 164 \\
\hline & 0651330501 & $2.20 \pm 0.18$ & $0.13 \pm 0.01$ & $1.84 \pm 0.10$ & - & 1.11 & 153 \\
\hline & 0109080801 & $2.24 \pm 0.08$ & $0.11 \pm 0.01$ & $1.87 \pm 0.05$ & - & 0.88 & 191 \\
\hline & 0651330701 & $2.24 \pm 0.07$ & $0.12 \pm 0.01$ & $1.70 \pm 0.08$ & - & 0.98 & 171 \\
\hline & 0651331101 & $2.21 \pm 0.02$ & $0.11 \pm 0.01$ & $1.60 \pm 0.05$ & - & 1.09 & 151 \\
\hline \multirow[t]{5}{*}{ PG1116+215 } & 0201940101 & $2.85 \pm 0.04$ & $0.35 \pm 0.03$ & $1.92 \pm 0.05$ & $3.3 \pm 0.4$ & 1.34 & 225 \\
\hline & 0201940201 & $2.74 \pm 0.07$ & $0.30 \pm 0.06$ & $2.09 \pm 0.13$ & $1.0 \pm 0.5$ & 1.06 & 109 \\
\hline & 0554380201 & $2.87 \pm 0.02$ & $0.30 \pm 0.01$ & $1.84 \pm 0.03$ & $3.6 \pm 0.1$ & 1.27 & 218 \\
\hline & 0554380301 & $2.99 \pm 0.03$ & $0.35 \pm 0.01$ & $1.85 \pm 0.04$ & $4.3 \pm 0.4$ & 1.24 & 209 \\
\hline & 0554380101 & $2.86 \pm 0.03$ & $0.44 \pm 0.06$ & $1.84 \pm 0.03$ & $4.0 \pm 0.3$ & 1.10 & 218 \\
\hline \multirow[t]{3}{*}{ PG1351+640 } & 0205390301 & $2.78 \pm 0.09$ & $0.24 \pm 0.04$ & $2.01 \pm 0.12$ & $2.1 \pm 0.4$ & 1.11 & 130 \\
\hline & 0556230201 & $2.58 \pm 0.26$ & $0.15 \pm 0.03$ & $1.50 \pm 0.10$ & $2.2 \pm 1.1$ & 1.08 & 46 \\
\hline & 0556230101 & $2.24 \pm 0.19$ & $0.11 \pm 0.02$ & $1.50 \pm 0.08$ & $1.9 \pm 1.0$ & 1.79 & 25 \\
\hline \multirow[t]{2}{*}{ PG1402+261 } & 0400200201 & $2.90 \pm 0.22$ & $0.64 \pm 4.84$ & $1.86 \pm 0.32$ & $3.2 \pm 1.2$ & 1.08 & 112 \\
\hline & 0400200101 & $2.83 \pm 0.13$ & $0.31 \pm 0.10$ & $2.04 \pm 0.13$ & $3.4 \pm 0.9$ & 0.97 & 164 \\
\hline \multirow[t]{4}{*}{ PG1440+356 } & 0005010301 & $2.59 \pm 0.10$ & $0.18 \pm 0.03$ & $2.33 \pm 0.09$ & $2.1 \pm 0.8$ & 1.09 & 141 \\
\hline & 0107660201 & $2.51 \pm 0.10$ & $0.19 \pm 0.02$ & $2.39 \pm 0.08$ & $2.5 \pm 0.6$ & 0.78 & 169 \\
\hline & 0005010201 & $2.68 \pm 0.18$ & $0.21 \pm 0.07$ & $2.22 \pm 0.15$ & $3.6 \pm 1.2$ & 1.09 & 163 \\
\hline & 0005010101 & $2.65 \pm 0.19$ & $0.20 \pm 0.05$ & $2.29 \pm 0.09$ & $3.5 \pm 1.4$ & 1.30 & 174 \\
\hline \multirow[t]{3}{*}{ Q0056-363 } & 0205680101 & $2.71 \pm 0.06$ & $1.83 \pm 4.58$ & $1.51 \pm 0.12$ & $3.2 \pm 0.4$ & 1.29 & 216 \\
\hline & 0401930101 & $2.62 \pm 0.09$ & $0.34 \pm 0.08$ & $1.95 \pm 0.07$ & $2.0 \pm 0.6$ & 0.98 & 191 \\
\hline & 0102040701 & $2.68 \pm 0.10$ & $0.32 \pm 0.09$ & $2.08 \pm 0.11$ & $1.0 \pm 0.7$ & 0.85 & 146 \\
\hline \multirow[t]{8}{*}{ RE1034+396 } & 0506440101 & $2.82 \pm 0.05$ & $0.19 \pm 0.01$ & $2.39 \pm 0.04$ & $12.9 \pm 0.5$ & 1.90 & 169 \\
\hline & 0675440201 & $3.20 \pm 0.11$ & $0.23 \pm 0.03$ & $2.01 \pm 0.20$ & $20.0 \pm 0.5$ & 0.83 & 117 \\
\hline & 0675440101 & $3.16 \pm 0.05$ & $0.21 \pm 0.02$ & $2.12 \pm 0.18$ & $20.0 \pm 0.3$ & 1.16 & 117 \\
\hline & 0109070101 & $3.02 \pm 0.10$ & $0.21 \pm 0.03$ & $2.28 \pm 0.13$ & $17.9 \pm 1.4$ & 1.38 & 90 \\
\hline & 0655310101 & $3.14 \pm 0.08$ & $0.20 \pm 0.02$ & $2.26 \pm 0.14$ & $19.7 \pm 0.7$ & 1.01 & 121 \\
\hline & 0675440301 & $2.50 \pm 0.08$ & $0.16 \pm 0.01$ & $2.11 \pm 0.12$ & $14.3 \pm 1.4$ & 1.15 & 118 \\
\hline & 0561580201 & $2.61 \pm 0.07$ & $0.15 \pm 0.01$ & $2.35 \pm 0.11$ & $15.7 \pm 1.1$ & 1.14 & 130 \\
\hline & 0655310201 & $3.19 \pm 0.10$ & $0.22 \pm 0.04$ & $2.17 \pm 0.18$ & $19.3 \pm 0.9$ & 1.13 & 141 \\
\hline \multirow[t]{5}{*}{ UGC3973 } & 0103862101 & $2.21 \pm 0.06$ & $0.24 \pm 0.03$ & $1.84 \pm 0.10$ & $1.9^{f}$ & 1.16 & 160 \\
\hline & 0400070401 & $2.30 \pm 0.02$ & $0.17 \pm 0.01$ & $1.90 \pm 0.03$ & - & 1.35 & 219 \\
\hline & 0502091001 & $2.39 \pm 0.03$ & $0.19 \pm 0.01$ & $1.50 \pm 0.00$ & - & 2.36 & 225 \\
\hline & 0400070201 & $2.17 \pm 0.04$ & $0.16 \pm 0.01$ & $2.09 \pm 0.03$ & - & 1.32 & 223 \\
\hline & 0400070301 & $2.42 \pm 0.04$ & $0.37 \pm 0.07$ & $1.82 \pm 0.05$ & - & 1.09 & 219 \\
\hline
\end{tabular}


Table C.2. Bolometric luminosities (in Eddington units) for the different observations of the sample for which an estimation of the object black hole mass is known (see Table B.1).

\begin{tabular}{|c|c|c|}
\hline Source & ObsID & $L_{\mathrm{bol}} / L_{\mathrm{Edd}}$ \\
\hline \multirow[t]{9}{*}{ 1H0419-577 } & 0148000201 & 0.098 \\
\hline & 0148000301 & 0.097 \\
\hline & 0148000401 & 0.290 \\
\hline & 0148000501 & 0.166 \\
\hline & 0148000601 & 0.102 \\
\hline & 0604720301 & 0.156 \\
\hline & 0604720401 & 0.128 \\
\hline & 0112600401 & 0.195 \\
\hline & Average & 0.154 \\
\hline \multirow[t]{4}{*}{ ESO198-G24 } & 0305370101 & 0.012 \\
\hline & 0112910101 & 0.009 \\
\hline & 0067190101 & 0.013 \\
\hline & Average & 0.011 \\
\hline \multirow[t]{3}{*}{ HE1029-1401 } & 0203770101 & 0.102 \\
\hline & 0110950101 & 0.090 \\
\hline & Average & 0.096 \\
\hline \multirow[t]{3}{*}{ IRASF12397+3333 } & 0202180201 & 0.615 \\
\hline & 0202180301 & 0.270 \\
\hline & Average & 0.443 \\
\hline \multirow[t]{4}{*}{ MRK279 } & 0302480501 & 0.120 \\
\hline & 0302480601 & 0.121 \\
\hline & 0302480401 & 0.127 \\
\hline & Average & 0.123 \\
\hline \multirow[t]{4}{*}{ MRK335 } & 0600540601 & 0.172 \\
\hline & 0600540501 & 0.186 \\
\hline & 0510010701 & 0.167 \\
\hline & Average & 0.175 \\
\hline \multirow[t]{17}{*}{ MRK509 } & 0601390201 & 0.127 \\
\hline & 0601390301 & 0.123 \\
\hline & 0601390401 & 0.157 \\
\hline & 0601390501 & 0.176 \\
\hline & 0601390601 & 0.197 \\
\hline & 0601390701 & 0.157 \\
\hline & 0601390801 & 0.146 \\
\hline & 0601390901 & 0.131 \\
\hline & 0601391001 & 0.135 \\
\hline & 0601391101 & 0.134 \\
\hline & 0306090101 & 0.068 \\
\hline & 0306090201 & 0.093 \\
\hline & 0306090301 & 0.097 \\
\hline & 0306090401 & 0.092 \\
\hline & 0130720101 & 0.056 \\
\hline & 0130720201 & 0.077 \\
\hline & Average & 0.123 \\
\hline \multirow[t]{3}{*}{ MRK590 } & 0109130301 & 0.008 \\
\hline & 0201020201 & 0.009 \\
\hline & Average & 0.008 \\
\hline \multirow[t]{5}{*}{ MRK883 } & 0652550201 & 0.044 \\
\hline & 0302260701 & 0.036 \\
\hline & 0302261001 & 0.039 \\
\hline & 0302260101 & 0.035 \\
\hline & Average & 0.038 \\
\hline \multirow[t]{4}{*}{ NGC4593 } & 0740920201 & 0.046 \\
\hline & 0740920301 & 0.025 \\
\hline & 0740920401 & 0.029 \\
\hline & 0740920501 & 0.044 \\
\hline
\end{tabular}

Notes. We also list the average bolometric luminosities for each object.
Table C.2. Continued

\begin{tabular}{|c|c|c|}
\hline Source & ObsID & $L_{\mathrm{bol}} / L_{\mathrm{Edd}}$ \\
\hline \multirow{7}{*}{ PG0804+761 } & 0740920601 & 0.043 \\
\hline & 0109970101 & 0.053 \\
\hline & 0059830101 & 0.075 \\
\hline & Average & 0.045 \\
\hline & 0605110101 & 0.402 \\
\hline & 0605110201 & 0.434 \\
\hline & Average & 0.418 \\
\hline \multirow[t]{3}{*}{ PG0844+349 } & 0554710101 & 0.085 \\
\hline & 0103660201 & 0.107 \\
\hline & Average & 0.096 \\
\hline \multirow{13}{*}{ PG1114+445 } & 0651330201 & 0.072 \\
\hline & 0651330101 & 0.053 \\
\hline & 0651330301 & 0.056 \\
\hline & 0651330401 & 0.072 \\
\hline & 0651330601 & 0.064 \\
\hline & 0651330901 & 0.050 \\
\hline & 0651330801 & 0.047 \\
\hline & 0651331001 & 0.044 \\
\hline & 0651330501 & 0.061 \\
\hline & 0109080801 & 0.046 \\
\hline & 0651330701 & 0.053 \\
\hline & 0651331101 & 0.056 \\
\hline & Average & 0.056 \\
\hline \multirow[t]{6}{*}{ PG1116+215 } & 0201940101 & 0.384 \\
\hline & 0201940201 & 0.392 \\
\hline & 0554380201 & 0.373 \\
\hline & 0554380301 & 0.425 \\
\hline & 0554380101 & 0.404 \\
\hline & Average & 0.395 \\
\hline \multirow[t]{4}{*}{ PG1351+640 } & 0205390301 & 0.269 \\
\hline & 0556230201 & 0.238 \\
\hline & 0556230101 & 0.296 \\
\hline & Average & 0.268 \\
\hline \multirow[t]{3}{*}{ PG1402+261 } & 0400200201 & 0.438 \\
\hline & 0400200101 & 0.485 \\
\hline & Average & 0.461 \\
\hline \multirow[t]{5}{*}{ PG1440+356 } & 0005010301 & 0.458 \\
\hline & 0107660201 & 0.635 \\
\hline & 0005010201 & 0.625 \\
\hline & 0005010101 & 0.635 \\
\hline & Average & 0.588 \\
\hline \multirow[t]{4}{*}{ Q0056-363 } & 0205680101 & 0.053 \\
\hline & 0401930101 & 0.041 \\
\hline & 0102040701 & 0.073 \\
\hline & Average & 0.056 \\
\hline \multirow[t]{9}{*}{ RE1034+396 } & 0506440101 & 1.500 \\
\hline & 0675440201 & 1.819 \\
\hline & 0675440101 & 1.889 \\
\hline & 0109070101 & 1.519 \\
\hline & 0655310101 & 1.804 \\
\hline & 0675440301 & 1.691 \\
\hline & 0561580201 & 1.698 \\
\hline & 0655310201 & 1.750 \\
\hline & Average & 1.709 \\
\hline \multirow[t]{6}{*}{ UGC3973 } & 0103862101 & 0.027 \\
\hline & 0400070401 & 0.028 \\
\hline & 0502091001 & 0.025 \\
\hline & 0400070201 & 0.034 \\
\hline & 0400070301 & 0.025 \\
\hline & Average & 0.028 \\
\hline
\end{tabular}


P.-O. Petrucci et al.: Testing warm Comptonization models for the origin of the soft X-ray excess in AGNs

\section{Appendix D: Particular model fitting procedure}

For 13 objects of our sample, HB890405-123, LBQS1228+1116, MRK 279, MRK 883, PG0804+761, PG0844+349, PG1116+215, PG1351+640, PG1402+261, PG1440+356, Q0056-363, RE1034+396, and PG1114+445, the fitting procedure explained in Sect. 4.2.2 gives good fits. For the other objects, however, additional model components significantly improve the fit. For IRASF12397+3333, MRK509, MRK590, ESO198-G24, HE1029-1401, NGC4593, and UGC3973, we add two narrow Gaussian lines to fit the strong residuals of emission/absorption line-like features below $2 \mathrm{keV}$. For 1H0419-577 and MRK335, we add a second CLOUDY table to fit the residual WA features. In the case of PG1114+445, ESO198-G24, HE1029-1401, NGC4593, and UGC3973, the disk temperature is also fixed during the fitting procedure. 